\title{
Non-intersecting squared Bessel paths with one positive starting and ending point
}

\author{
Steven Delvaux, Arno B. J. Kuijlaars*, Pablo Román*†and Lun Zhang* \\ Published in \\ Journal d'Analyse Mathématique 118 (2012), 105-159.
}

\begin{abstract}
We consider a model of $n$ non-intersecting squared Bessel processes with one starting point $a>0$ at time $t=0$ and one ending point $b>0$ at time $t=T$. After proper scaling, the paths fill out a region in the $t x$-plane. Depending on the value of the product $a b$ the region may come to the hard edge at 0 , or not. We formulate a vector equilibrium problem for this model, which is defined for three measures, with upper constraints on the first and third measures and an external field on the second measure. It is shown that the limiting mean distribution of the paths at time $t$ is given by the second component of the vector that minimizes this vector equilibrium problem. The proof is based on a steepest descent analysis for a $4 \times 4$ matrix valued Riemann-Hilbert problem which characterizes the correlation kernel of the paths at time $t$. We also discuss the precise locations of the phase transitions.
\end{abstract}

\section{Introduction}

Let $X(t)$ be a squared Bessel process with parameter $\alpha>-1$, i.e., it is a diffusion process (a strong Markov process with continuous sample paths) on $\mathbb{R}^{+}$with transition probability density given by (cf. $[11,36])$

$$
\begin{aligned}
p_{t}^{\alpha}(x, y) & =\frac{1}{2 t}\left(\frac{y}{x}\right)^{\alpha / 2} e^{-\frac{x+y}{2 t}} I_{\alpha}\left(\frac{\sqrt{x y}}{t}\right), & x, y>0, \\
p_{t}^{\alpha}(0, y)=\frac{y^{\alpha}}{(2 t)^{\alpha+1} \Gamma(\alpha+1)} e^{-\frac{y}{2 t}}, & y & >0,
\end{aligned}
$$

where

$$
I_{\alpha}(z)=\sum_{k=0}^{\infty} \frac{(z / 2)^{2 k+\alpha}}{k ! \Gamma(k+\alpha+1)}
$$

${ }^{*}$ Department of Mathematics, Katholieke Universiteit Leuven, Celestijnenlaan 200B, B-3001 Leuven, Belgium. E-mail: \{steven.delvaux,arno.kuijlaars,pablo.roman,lun.zhang\}@wis.kuleuven.be.

${ }^{\dagger}$ CIEM, FaMAF, Universidad Nacional de Córdoba, Medina Allende s/n Ciudad Universitaria, Córdoba, Argentina. E-mail: roman@famaf.unc.edu.ar. 
is the modified Bessel function of the first kind of order $\alpha$; see [1, p.375]. If $d=2(\alpha+1)$ is an integer, the squared Bessel process can be viewed as the square of the distance to the origin of a $d$-dimensional Brownian motion. Some applications of this diffusion process can be found, for example, in [30].

We consider a model of $n$ independent copies $X_{j}(t), j=1, \ldots, n$, conditioned such that for some $T>0$,

- $X_{j}(0)=a_{j}, X_{j}(T)=b_{j}$ for $j=1, \ldots, n$ for some given values $0 \leq a_{1}<a_{2}<\cdots<$ $a_{n}, 0 \leq b_{1}<b_{2}<\cdots<b_{n}$,

- the paths do not intersect each other for $0<t<T$.

It follows from the seminal paper of Karlin and McGregor [31] that the positions of the non-intersecting squared Bessel paths at any given time $t \in(0, T)$ are a determinantal point process. This means that there exists a correlation kernel $K_{n}$ such that for each positive integer $m$ the $m$-point correlation function takes the determinantal form

$$
\operatorname{det}\left[K_{n}\left(x_{j}, x_{k}\right)\right]_{j, k=1, \ldots, m} .
$$

Indeed, we have by [31] that the positions of the paths at time $t$ have a joint probability density

$$
\mathcal{P}_{n, t}\left(x_{1}, \ldots, x_{n}\right)=\frac{1}{Z_{n, t}} \operatorname{det}\left[f_{j}\left(x_{k}\right)\right]_{j, k=1, \ldots, n} \operatorname{det}\left[g_{j}\left(x_{k}\right)\right]_{j, k=1, \ldots, n},
$$

where $f_{j}(x)=p_{t}^{\alpha}\left(a_{j}, x\right), g_{j}(x)=p_{T-t}^{\alpha}\left(x, b_{j}\right), j=1, \ldots, n$, and $Z_{n, t}$ is the normalization constant

$$
Z_{n, t}=\int_{(0, \infty)^{n}} \mathcal{P}_{n, t}\left(x_{1}, \ldots, x_{n}\right) d x_{1} \ldots d x_{n} .
$$

Hence, (1.5) is a biorthogonal ensemble [9], which is a special case of a determinantal point process. The correlation kernel is given by

$$
K_{n}(x, y)=\sum_{j=1}^{n} \phi_{j}(x) \psi_{j}(y),
$$

where the functions $\phi_{j}, \psi_{j}, j=1, \ldots, n$ are such that

$$
\operatorname{span}\left(\phi_{1}, \ldots, \phi_{n}\right)=\operatorname{span}\left(f_{1}, \ldots, f_{n}\right), \quad \operatorname{span}\left(\psi_{1}, \ldots, \psi_{n}\right)=\operatorname{span}\left(g_{1}, \ldots, g_{n}\right),
$$

and

$$
\int_{0}^{\infty} \phi_{i}(x) \psi_{k}(x) d x=\delta_{i, k}, \quad i, k=1, \ldots, n .
$$

We are interested in the situation where all paths start at the same position at $t=0$ and end at the same position at $t=T$. Different types of initial and ending conditions are considered in $[32,34,35]$, among others. In the confluent limit $a_{j} \rightarrow a \geq 0$ and $b_{j} \rightarrow b \geq 0$, the biorthogonal ensemble structure is preserved. 

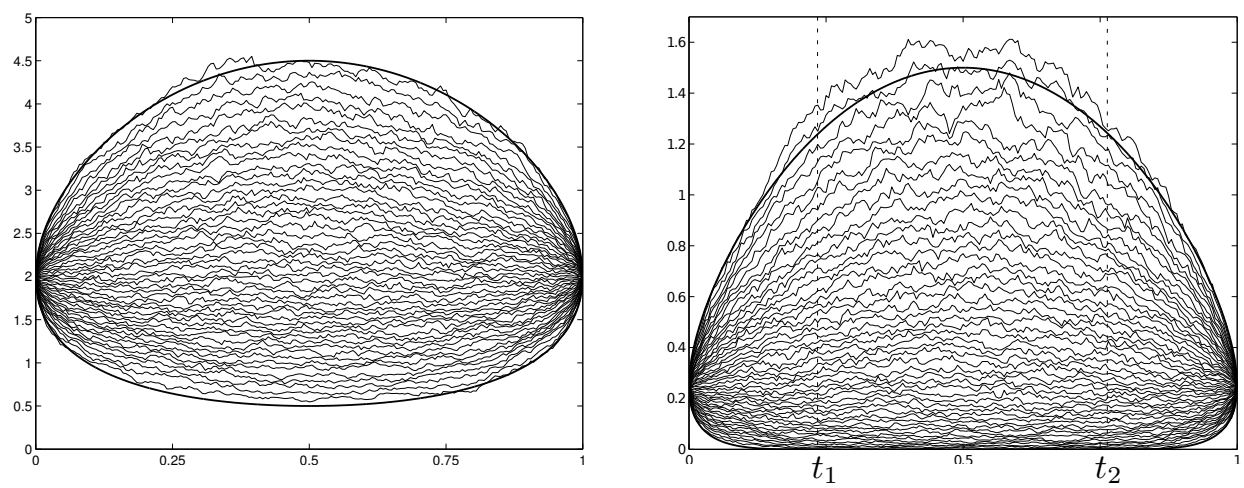

Figure 1: 50 rescaled non-intersecting squared Bessel paths with $a=b=2$ (left) and $a=b=1 / 4$ (right).

If $a=b=0$, i.e., all paths start and end at the origin, this system is known as chiral or Laguerre ensemble in random matrix theory, cf. [20, 33, 36, 49]. The case where $a>0$ and $b=0$ was considered in [38] and [39]. In that situation, all paths, after proper scaling, initially stay away from the hard edge at $x=0$, but at a certain critical time $t^{*}$ the lowest paths hit the hard edge and are stuck to it from then on. The limiting mean density of the paths at time $t$ is characterized by a non-real solution of an algebraic equation of order three; see also [38, Appendix] and [42] for an interpretation in terms of a vector equilibrium problem with two measures. If $t \neq t^{*}$, the local correlations obey the usual scaling limit from the random matrix theory, leading to the sine, Airy, and Bessel kernels [38]. A new kernel was established near the origin at the critical time $t^{*}$ in [39]. The kernel admits a double integral representation which resembles the Pearcey kernel $[8,10,12]$.

In this paper, we consider the case where both $a$ and $b$ are positive. To obtain interesting results, we make a time scaling as in [38] so that the time variable depends on the number of paths $n$. That is, we replace the variables $t$ and $T$ by

$$
t \mapsto \frac{t}{2 n}, \quad T \mapsto \frac{1}{2 n},
$$

so that $0<t<1$. Now, letting $n \rightarrow \infty$, the paths fill out a region in the $t x$-plane that looks like one of the regions shown in Figures 1 and 2, depending on the product $a b$. Suppose $a$ and $b$ stay far away from the hard edge $x=0$, such that $a b>1 / 4$. Then we are in a situation as illustrated by the left picture in Figure 1. All paths remain positive for all time $t$. On the other hand, if $a b<1 / 4$, there are two critical times $t_{1}$ and $t_{2}$, as depicted in the right picture of Figure 1. All paths initially stay away from the hard edge (a wall) and the lowest paths hit the hard edge at the time $t_{1}$. Since all paths are required to end at a positive position $b$, the lowest paths are stuck to the hard edge only for a while, and they leave the wall at a second critical time $t_{2}$. The times $t_{1}$ and $t_{2}$ will come together if $a b=1 / 4$, and we are then led to an intermediate situation shown in the left picture in Figure 2. The paths fill out a region bounded by the solid line, which 

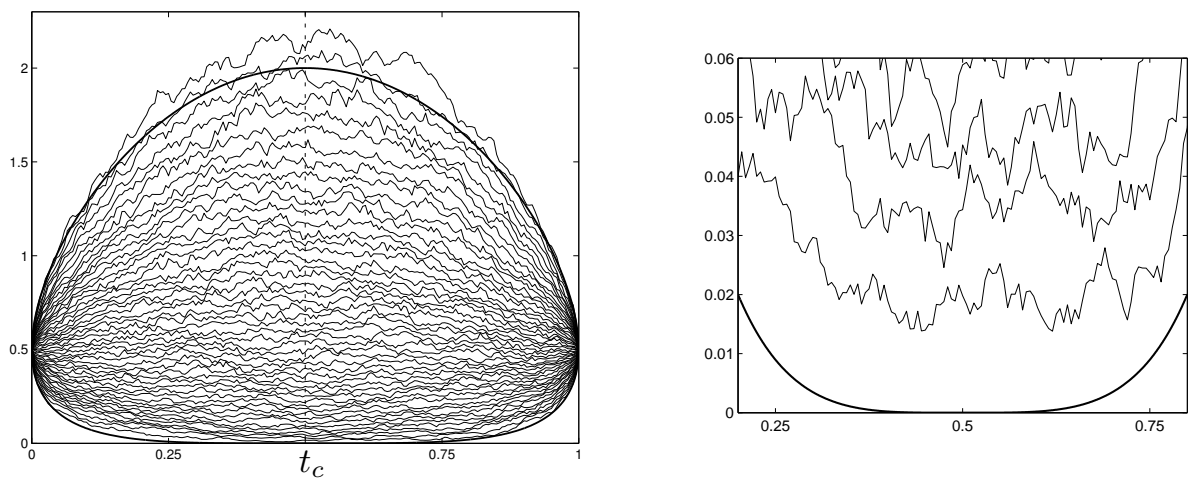

Figure 2: 50 rescaled non-intersecting squared Bessel paths with $a=b=1 / 2$ and $t_{c}=1 / 2$ (left) and around the point $t_{c}$ (right).

is tangent to the hard edge at a multicritical time $t_{c}$; see also the right picture in Figure 2 for a magnified view around $t_{c}$.

It is the aim of this paper to describe the region that is filled in the large $n$ limit by the non-intersecting squared Bessel paths with one positive starting point and one positive ending point. Our results are stated in the next section.

\section{Statement of results}

\subsection{A vector equilibrium problem}

The main ingredient of our analysis is a vector equilibrium problem involving both an external field and two upper constraints. To this end, we define, as usual (see [48]), the logarithmic energy of a measure $\mu$ by

$$
I(\mu)=\iint \log \frac{1}{|x-y|} d \mu(x) d \mu(y),
$$

and the mutual energy of two measures $\mu, \nu$ by

$$
I(\mu, \nu)=\iint \log \frac{1}{|x-y|} d \mu(x) d \nu(y) .
$$

Consider the energy functional

$$
E\left(\nu_{1}, \nu_{2}, \nu_{3}\right)=\sum_{j=1}^{3} I\left(\nu_{j}\right)-\sum_{j=1}^{2} I\left(\nu_{j}, \nu_{j+1}\right)+\int V(x) d \nu_{2}(x),
$$

where

$$
V(x)=\frac{x}{t(1-t)}-\frac{2 \sqrt{a x}}{t}-\frac{2 \sqrt{b x}}{1-t} .
$$

The vector equilibrium problem is to minimize $E\left(\nu_{1}, \nu_{2}, \nu_{3}\right)$ among all measures $\nu_{1}$, $\nu_{2}$ and $\nu_{3}$ satisfying the following conditions (E1)-(E3): 
(E1) $\nu_{1}$ is a measure on $\mathbb{R}^{-}:=(-\infty, 0]$ with total mass $1 / 2$ that satisfies the constraint

$$
\nu_{1} \leq \rho_{1},
$$

where $\rho_{1}$ is the measure on $\mathbb{R}^{-}$with density

$$
\frac{d \rho_{1}}{d x}=\frac{\sqrt{a}}{\pi t}|x|^{-1 / 2}, \quad x \in \mathbb{R}^{-} .
$$

(E2) $\nu_{2}$ is a measure on $\mathbb{R}^{+}:=[0, \infty)$ with total mass 1 .

(E3) $\nu_{3}$ is a measure on $\mathbb{R}^{-}$with total mass $1 / 2$ that satisfies the constraint

$$
\nu_{3} \leq \rho_{3},
$$

where $\rho_{3}$ is the measure on $\mathbb{R}^{-}$with density

$$
\frac{d \rho_{3}}{d x}=\frac{\sqrt{b}}{\pi(1-t)}|x|^{-1 / 2}, \quad x \in \mathbb{R}^{-} .
$$

An electrostatic interpretation of the above equilibrium problem is the following: Consider three types of charged particles. The particles of the first and the third types are put on $\mathbb{R}^{-}$, both having total charge $\frac{1}{2}$. The particles of the second type are put on $\mathbb{R}^{+}$with total charge 1. Particles of the same type repel each other. The particles of the first and the second types interact with each other with an attraction that is only half of the strength of the repulsion of particles of the same type. So do the particles of the second and the third types. The particles of the first and the third types do not interact directly. The particles of the second type are influenced by the external field $V$. The measures $\rho_{1}$ and $\rho_{3}$ in (2.6) and (2.8) play the role of upper constraints for the first and the third measures. Namely, we minimize the equilibrium problem with the extra condition that the particle densities of the first and the third types do not exceed the densities of $\rho_{1}$ and $\rho_{3}$, respectively.

Vector equilibrium problems with mutual interaction can be traced back to [27, 28], see also [46]. The equilibrium problem with constraints appeared before mainly in the asymptotic analysis of discrete orthogonal polynomials, see e.g. [6, 21, 47].

Our first result concerns the structure of the minimizer of the vector equilibrium problem.

Theorem 2.1. Let $a>0, b>0$ and $0<t<1$. Then there is a unique minimizer $\left(\mu_{1}, \mu_{2}, \mu_{3}\right)$ of the energy functional (2.3) under the conditions (E1), (E2), (E3). The minimizing measures satisfy:

(a) The support of $\mu_{1}$ is the negative real axis and there exists $r_{1} \geq 0$ such that

$$
\operatorname{supp}\left(\rho_{1}-\mu_{1}\right)=\left(-\infty,-r_{1}\right] .
$$

The density of the measure $\rho_{1}-\mu_{1}$ vanishes like a square root at $-r_{1}$ if $r_{1}>0$. 
(b) The support of $\mu_{2}$ is bounded and there exist $0 \leq p<q$ such that

$$
\operatorname{supp}\left(\mu_{2}\right)=[p, q]
$$

and $\mu_{2}$ has a density which is real analytic in the interior of the support and vanishes like a square root at the endpoint $q$ and at $p$ if $p>0$.

(c) The support of $\mu_{3}$ is the negative real axis and there exists $r_{3} \geq 0$ such that

$$
\operatorname{supp}\left(\rho_{3}-\mu_{3}\right)=\left(-\infty,-r_{3}\right] .
$$

The density of the measure $\rho_{3}-\mu_{3}$ vanishes as a square root at $-r_{3}$ if $r_{3}>0$.

Note that $p, q, r_{1}$, and $r_{3}$ depend on $a, b$ and $t$.

The proof of Theorem 2.1 will be given in Section 3. Throughout the whole paper, we will denote

$$
\Delta_{1}:=\left(-\infty,-r_{1}\right), \quad \Delta_{2}:=(p, q), \quad \text { and } \quad \Delta_{3}:=\left(-\infty,-r_{3}\right),
$$

i.e., $\Delta_{j}, j=1,2,3$ is the interior of the $\operatorname{sets} \operatorname{supp}\left(\rho_{1}-\mu_{1}\right), \operatorname{supp}\left(\mu_{2}\right)$ and $\operatorname{supp}\left(\rho_{3}-\mu_{3}\right)$, respectively.

\subsection{Classification into cases}

It turns out that at least one of $r_{1}, p, r_{3}$ is equal to 0 , and generically no two consecutive ones are 0 . Generically we also have a $O\left(|x|^{-1 / 2}\right)$ behavior of the density at the hard edge 0 . Then there are three generic cases.

Case I $r_{1}=r_{3}=0$ and $p>0$. The densities of $\rho_{1}-\mu_{1}$ and $\rho_{3}-\mu_{3}$ blow up like $O\left(|x|^{-1 / 2}\right)$ as $x \rightarrow 0-$.

Case II $p>0$ and one of $r_{1}, r_{3}$ is zero and the other is positive. Thus there are two subcases which play a symmetric role.

subcase IIa $r_{1}>0, p>0$ and $r_{3}=0$.

subcase IIb $r_{1}=0, p>0$ and $r_{3}>0$.

Case III $r_{1}>0, p=0$ and $r_{3}>0$.

In Case I we have to add the condition that the densities of $\rho_{1}-\mu_{1}$ and $\rho_{3}-\mu_{3}$ blow up like an inverse square root at 0 in order to be in a generic case. There is also a non-generic case where one of these densities vanishes as a square root. This is exactly what happens in the transition from Case I to Case II. See Case V below.

The non-generic cases are:

Case IV Exactly two consecutive values from $r_{1}, p, r_{2}$ are zero. Thus $p=0$ and one of $r_{1}, r_{3}$ is also zero, while the other is positive. 
Case $\mathbf{V} r_{1}=r_{3}=0$ and $p>0$. One of the densities of $\rho_{j}-\mu_{j}$ vanishes like a square root $O\left(|x|^{1 / 2}\right)$ as $x \rightarrow 0-$, while the other blows up like $O\left(|x|^{-1 / 2}\right)$ as $x \rightarrow 0-$.

Case VI All three are zero: $r_{1}=p=r_{3}=0$.

The Case I corresponds to the situation as shown by the left picture in Figure 1. In this case $a b>1 / 4$ and all paths stay away from the hard edge at $x=0$ for all $t \in(0,1)$. Both constraints are never active.

The right picture of Figure 1 which deals with $a b<1 / 4$, contains the three Cases II, III, IV. If $t<t_{1}$ or $t>t_{2}$, then we are in Case II, where only one constraint is active. If $t_{1}<t<t_{2}$, then we are then in Case III, where both constraints are active. The times $t_{1}$ and $t_{2}$ correspond to Case IV, which represents a phase transition between soft edge and hard edge.

The final Cases V and VI are contained in Figure 2 for the situation $a b=1 / 4$ when the domain just touches the wall. We are in Case V if $t \neq t_{c}$, and in case VI if $t=t_{c}$.

The Cases IV-VI are non-generic critical cases. They correspond to certain phase transitions between the generic cases; see also Section 4.4 for a phase diagram in the situation where $a b<1 / 4$.

We shall only consider the generic cases in this paper. For convenience (and without loss of generality), it is assumed that

$$
r_{3} \geq r_{1}
$$

in Cases II and III, which implies that $\Delta_{3} \subset \Delta_{1} \subset \mathbb{R}^{-}$holds in all three cases.

\subsection{Variational conditions}

Denote by

$$
U^{\mu}(x)=\int \log \frac{1}{|x-y|} d \mu(y)
$$

the logarithmic potential of a measure $\mu$. The minimizer $\left(\mu_{1}, \mu_{2}, \mu_{3}\right)$ is characterized by the following Euler-Lagrange variational conditions:

Proposition 2.2. The measures $\mu_{1}, \mu_{2}$ and $\mu_{3}$ satisfy for some constant $l \in \mathbb{R}$,

$$
\begin{array}{ll}
2 U^{\mu_{2}}(x)=U^{\mu_{1}}(x)+U^{\mu_{3}}(x)-V(x)+l, & x \in \operatorname{supp}\left(\mu_{2}\right), \\
2 U^{\mu_{2}}(x)>U^{\mu_{1}}(x)+U^{\mu_{3}}(x)-V(x)+l, & x \in \mathbb{R}^{+} \backslash \operatorname{supp}\left(\mu_{2}\right),
\end{array}
$$

where $V(x)$ is given in (2.4);

$$
\begin{array}{ll}
2 U^{\mu_{1}}(x)=U^{\mu_{2}}(x), & x \in \operatorname{supp}\left(\rho_{1}-\mu_{1}\right), \\
2 U^{\mu_{1}}(x)<U^{\mu_{2}}(x), & x \in \mathbb{C} \backslash \operatorname{supp}\left(\rho_{1}-\mu_{1}\right),
\end{array}
$$

and

$$
\begin{array}{ll}
2 U^{\mu_{3}}(x)=U^{\mu_{2}}(x), & x \in \operatorname{supp}\left(\rho_{3}-\mu_{3}\right), \\
2 U^{\mu_{3}}(x)<U^{\mu_{2}}(x), & x \in \mathbb{C} \backslash \operatorname{supp}\left(\rho_{3}-\mu_{3}\right) .
\end{array}
$$


Note that the inequalities in (2.16), (2.18) and (2.20) are all strict. The strictness of the inequality in (2.16) follows from the strict convexity of the external fields acting the second measure; see Lemma 3.3 below.

The proof of Proposition 2.2 will be given in Section 3.

\subsection{Limiting mean distribution}

The following is the main theorem of this paper.

Theorem 2.3. Let $a, b>0,0<t<1$, and let $K_{n}$ denote the correlation kernel for the positions of the non-intersecting squared Bessel paths at time $t$ in the rescaled time variables (1.9). Let $\left(\mu_{1}, \mu_{2}, \mu_{3}\right)$ be the minimizer of the vector equilibrium problem stated in Section 2.1. Suppose that we are in one of the generic cases I, II, or III, described above, then

$$
\lim _{n \rightarrow \infty} \frac{1}{n} K_{n}(x, x)=\frac{d \mu_{2}}{d x}(x), \quad x \in \mathbb{R}^{+} .
$$

The limit (2.21) expresses that the density of the measure $\mu_{2}$ is the limiting mean density of the non-intersecting squared Bessel paths at time $t$ as $n \rightarrow \infty$.

It is an unusual feature that it is the second measure which plays the main role here. In recent papers $[23,24]$ concerning eigenvalue distribution of a two-matrix model in random matrix theory, a vector equilibrium problem with three measures appears as well. In that situation, however, the first component of the minimizer is relevant to the limiting mean distribution.

We prove Theorem 2.3 by means of a steepest descent analysis of a relevant RiemannHilbert problem. From this analysis, we also obtain the universality results for the local path correlations. For Cases I, II, III, we obtain the sine kernel (in the bulk), Airy kernel (at the soft edges) and Bessel kernel of order $\alpha$ (at the hard edge 0), which are the typical limiting kernels in random matrix theory, see e.g. [15, 26, 44]. We will not discuss this any further but instead refer to the papers $[4,7,8,23]$ for a more detailed analysis in similar situations.

We expect the conclusion of Theorem 2.3 to remain valid in the Cases IV-VI. The local path correlations at the origin in Case IV should be the same as those derived in [39], since they both correspond to phase transitions between a soft and a hard edge. The Cases V-VI represent new critical phenomena. It is conjectured that they are related to the inhomogeneous Painlevé II equation, which bears some connections with the "critical separation' case for non-intersecting Brownian motion with two starting points and two ending points $[2,18,19,22]$. These results will be reported in a future publication.

Theorem 2.3 will be proved in Section 6 .

\subsection{About the proof of Theorem 2.3}

The proof of Theorem 2.3 relies on the Riemann-Hilbert $(\mathrm{RH})$ problem for multiple orthogonal polynomials of mixed type and its connection to the correlation kernel (1.7). To see this, we start with a proposition which gives us the explicit structure of the biorthogonal ensemble in the confluent case. 
Proposition 2.4. In confluent case $a_{j} \rightarrow a>0, b_{j} \rightarrow b>0, j=1, \ldots, n$, the positions of the non-intersecting squared Bessel paths at time $t \in(0, T)$ are a biorthogonal ensemble (1.5) with functions

$$
\begin{aligned}
f_{2 j-1}(x) & =x^{j-1} p_{t}^{\alpha}(a, x), & & j=1, \ldots, n_{1}:=\lceil n / 2\rceil, \\
f_{2 j}(x) & =x^{j-1} p_{t}^{\alpha+1}(a, x), & & j=1, \ldots, n_{2}:=n-n_{1}, \\
g_{2 j-1}(x) & =x^{j-1} p_{T-t}^{\alpha}(x, b), & j & =1, \ldots, n_{1}, \\
g_{2 j}(x) & =x^{j-1} p_{T-t}^{\alpha-1}(x, b), & j & =1, \ldots, n_{2} .
\end{aligned}
$$

Proof. The functions (2.22) and (2.23) follow as in the proof of Proposition 2.1 in [38].

In the confluent limit $b_{j} \rightarrow b$, the linear space spanned by the functions $x \mapsto$ $p_{T-t}^{\alpha}\left(x, b_{j}\right), j=1, \ldots, n$, tends to the linear space spanned by

$$
x \mapsto \frac{\partial^{j-1}}{\partial y^{j-1}} p_{T-t}^{\alpha}(x, b), \quad j=1, \ldots, n .
$$

Using the differential relations satisfied by the modified Bessel functions of the first kind (see [1]), we can prove that

$$
\begin{aligned}
\frac{\partial}{\partial y} p_{t}^{\alpha}(x, y) & =\frac{1}{2 t}\left(p_{t}^{\alpha-1}(x, y)-p_{t}^{\alpha}(x, y)\right) \\
y \frac{\partial}{\partial y} p_{t}^{\alpha-1}(x, y) & =\frac{x}{2 t} p_{t}^{\alpha}(x, y)-\left(\frac{y}{2 t}-(\alpha-1)\right) p_{t}^{\alpha-1}(x, y) .
\end{aligned}
$$

Now it is easy to show inductively that the linear span of (2.26) is the same as the linear space spanned by the functions (2.24) and (2.25).

Next, we introduce two sets of weight functions $w_{1,1}, w_{1,2}$ and $w_{2,1}, w_{2,2}$,

$$
\begin{array}{ll}
w_{1,1}(x)=x^{\alpha / 2} e^{-\frac{n x}{t}} I_{\alpha}\left(\frac{2 n \sqrt{a x}}{t}\right), & w_{1,2}(x)=x^{(\alpha+1) / 2} e^{-\frac{n x}{t}} I_{\alpha+1}\left(\frac{2 n \sqrt{a x}}{t}\right), \\
w_{2,1}(x)=x^{-\alpha / 2} e^{-\frac{n x}{1-t}} I_{\alpha}\left(\frac{2 n \sqrt{b x}}{1-t}\right), & w_{2,2}(x)=x^{-(\alpha-1) / 2} e^{-\frac{n x}{1-t}} I_{\alpha-1}\left(\frac{2 n \sqrt{b x}}{1-t}\right) .
\end{array}
$$

which are collected in two row vectors

$$
\mathbf{w}_{1}=\left(w_{1,1}, w_{1,2}\right), \quad \mathbf{w}_{2}=\left(w_{2,1}, w_{2,2}\right) .
$$

Note that by (1.1) and (1.9)

$$
\begin{array}{ll}
f_{2 j-1}(x) \propto x^{j-1} w_{1,1}(x), & f_{2 j}(x) \propto x^{j-1} w_{1,2}(x), \\
g_{2 j-1}(x) \propto x^{j-1} w_{2,1}(x), & g_{2 j}(x) \propto x^{j-1} w_{2,2}(x),
\end{array}
$$

where $f_{j}, g_{j}, j=1, \ldots, n$ are given in $(2.22)-(2.25)$. The proportionality constants are irrelevant for us.

We then look for a $4 \times 4$ matrix valued function $Y$ satisfying the following $\mathrm{RH}$ problem: 
(1) $Y$ is defined and analytic in $\mathbb{C} \backslash \mathbb{R}^{+}$.

(2) For $x>0, Y$ possesses continuous boundary values $Y_{+}(x)$ (from the upper half plane) and $Y_{-}(x)$ (from the lower half plane), which satisfy

$$
Y_{+}(x)=Y_{-}(x)\left(\begin{array}{cc}
I_{2} & W(x) \\
0 & I_{2}
\end{array}\right),
$$

where $I_{k}$ denotes the $k \times k$ identity matrix and $W(x)$ is the rank-one matrix

$$
\begin{aligned}
W(x) & =\mathbf{w}_{1}^{T}(x) \mathbf{w}_{2}(x) \\
& =e^{-\frac{n x}{(1-t) t}}\left(\begin{array}{cc}
I_{\alpha}\left(\frac{2 n \sqrt{a x}}{t}\right) I_{\alpha}\left(\frac{2 n \sqrt{b x}}{1-t}\right) & \sqrt{x} I_{\alpha}\left(\frac{2 n \sqrt{a x}}{t}\right) I_{\alpha-1}\left(\frac{2 n \sqrt{b x}}{1-t}\right) \\
\sqrt{x} I_{\alpha+1}\left(\frac{2 n \sqrt{a x}}{t}\right) I_{\alpha}\left(\frac{2 n \sqrt{b x}}{1-t}\right) & x I_{\alpha+1}\left(\frac{2 n \sqrt{a x}}{t}\right) I_{\alpha-1}\left(\frac{2 n \sqrt{b x}}{1-t}\right)
\end{array}\right) .
\end{aligned}
$$

(3) As $z \rightarrow \infty, z \in \mathbb{C} \backslash \mathbb{R}^{+}$, we have

$$
Y(z)=\left(I_{4}+O(1 / z)\right) \operatorname{diag}\left(z^{n_{1}}, z^{n_{2}}, z^{-n_{1}}, z^{-n_{2}}\right) .
$$

(4) $Y(z)$ has the following behavior near the origin:

$$
Y(z)=O\left(\begin{array}{cccc}
1 & 1 & h(z) & h(z) \\
1 & 1 & h(z) & h(z) \\
1 & 1 & h(z) & h(z) \\
1 & 1 & h(z) & h(z)
\end{array}\right), \quad Y(z)^{-1}=O\left(\begin{array}{cccc}
h(z) & h(z) & h(z) & h(z) \\
1 & 1 & 1 & 1 \\
1 & 1 & 1 & 1 \\
1 & 1 & 1 & 1
\end{array}\right)
$$

as $z \rightarrow 0, z \in \mathbb{C} \backslash \mathbb{R}^{+}$, where

$$
h(z)= \begin{cases}|z|^{\alpha}, & \text { if }-1<\alpha<0, \\ \log |z|, & \text { if } \alpha=0, \\ 1, & \text { if } \alpha>0\end{cases}
$$

and the $O$ condition in (2.32) is taken to be entrywise.

There exists a unique solution $Y$ to the above $\mathrm{RH}$ problem which involves multiple orthogonal polynomials of mixed type associated with the modified Bessel weights $\mathbf{w}_{1}(x)$, $\mathbf{w}_{2}(x)$ and their Cauchy transforms; see [13] for details. Due to the singularity of the weight matrix near the origin, we need the condition (2.32) to ensure the uniqueness of the solution. 
It also follows from the results in [13] that the correlation kernel (1.7) admits the following representation in terms of the solution of the $\mathrm{RH}$ problem:

$$
K_{n}(x, y)=\frac{1}{2 \pi i(x-y)}\left(\begin{array}{llll}
0 & 0 & w_{2,1}(y) & w_{2,2}(y)
\end{array}\right) Y_{+}^{-1}(y) Y_{+}(x)\left(\begin{array}{c}
w_{1,1}(x) \\
w_{1,2}(x) \\
0 \\
0
\end{array}\right)
$$

The representation (2.34) is based on the Christoffel-Darboux formula for multiple orthogonal polynomials of mixed type, see also [3].

We will obtain the limit (2.21) from a Deift/Zhou steepest descent analysis [15, 16, 17] for the $\mathrm{RH}$ problem for $Y$. It consists of a series of explicit and invertible transformations

$$
Y \mapsto X \mapsto U \mapsto T \mapsto R,
$$

which leads to a RH problem for a matrix valued function $R$ which is such that $R$ tends to the identity matrix as $n \rightarrow \infty$. Analyzing the effect of the transformations (2.35) we obtain the limit (2.21).

The rest of this paper is organized as follows. In Section 3 we analyze the equilibrium problem and establish Theorem 2.1 and Proposition 2.2. In Section 4 we introduce the Riemann surface built from the solution to the vector equilibrium problem and define some auxiliary functions. Section 5 contains the steepest descent analysis of the RH problem for $Y(z)$ and Section 6 gives the proof of the main Theorem 2.3. Throughout this paper, it is also assumed that $n$ is an even number so that

$$
n_{1}=n_{2}=n / 2 \text {. }
$$

This assumption is not essential and is only made to simplify the presentation.

\section{Analysis of the vector equilibrium problem}

The main purpose of this section is to prove Theorem 2.1 and Proposition 2.2, which yields the existence and main properties of the minimizers $\mu_{1}, \mu_{2}$ and $\mu_{3}$. Our basic tool is the balayage (sweeping out) of a measure.

For any finite measure $\nu$ we denote with $\|\nu\|$ the total mass of $\nu$. A positive measure $\hat{\nu}$ on a closed set $K \subset \mathbb{C}$ with positive capacity is called the balayage of $\nu$ onto $K$ if $\|\nu\|=\|\hat{\nu}\|$ and

$$
U^{\nu}(x)=U^{\hat{\nu}}(x)+C, \quad \text { q.e. } x \in K,
$$

for some constant $C$, where "q.e." means quasi-every, i.e., with the exception of a set with zero capacity, and $U^{\nu}$ is the logarithmic potential (2.14), see [48] for background on potential theory. To emphasize the dependence on $K$, we also write

$$
\hat{\nu}=\operatorname{Bal}(\nu, K)
$$


A relation between $\nu$ and $\operatorname{Bal}(\nu, K)$ is established as follows:

$$
\operatorname{Bal}(\nu, K)=\int \operatorname{Bal}\left(\delta_{s}, K\right) d \nu(s),
$$

where $\delta_{z}$ denotes the Dirac delta measure at the point $z$.

For the purpose of this paper, we are interested in the case where

$$
K=K_{c}=(-\infty,-c], \quad \text { with } c \geq 0 .
$$

Then $C=0$ in (3.1), since $K_{c}$ is unbounded. By using simple contour integration, it can be shown that, if $s \in \mathbb{R}^{+}$, the balayage of $\delta_{s}$ onto $K_{c}$ has the density

$$
\frac{d \operatorname{Bal}\left(\delta_{s}, K_{c}\right)}{d x}=\frac{1}{\pi} \frac{\sqrt{s+c}}{\sqrt{|x+c|}(s-x)}, \quad x \in K_{c} .
$$

In particular, for $c=0$,

$$
\frac{d \operatorname{Bal}\left(\delta_{s}, \mathbb{R}^{-}\right)}{d x}=\lim _{c \rightarrow 0+} \frac{d \operatorname{Bal}\left(\delta_{s}, K_{c}\right)}{d x}=\frac{1}{\pi} \frac{\sqrt{s}}{\sqrt{|x|}(s-x)}, \quad x \in \mathbb{R}^{-} .
$$

Observe that the density (3.4) is decreasing as $|x|$ increases, with a decay rate $O\left(|x|^{-3 / 2}\right)$ as $|x| \rightarrow \infty$. It will become clear in what follows how the concept of balayage measure is linked to the equilibrium problem.

\subsection{Equilibrium problems for $\nu_{1}$ and $\nu_{3}$}

If $\nu_{2}$ and $\nu_{3}$ are given, the equilibrium problem for $\nu_{1}$ is to minimize

$$
I(\nu)-\int U^{\nu_{2}}(x) d \nu(x)
$$

among all measures $\nu$ on $\mathbb{R}^{-}$with total mass $1 / 2$ and satisfying the constraint $\nu \leq \rho_{1}$, where $\rho_{1}$ is the measure on $\mathbb{R}^{-}$with density given by (2.6). Clearly, this problem only depends on $\nu_{2}$. The properties of $\nu_{1}$ are given in the following lemma.

Lemma 3.1. (The measure $\nu_{1}$ ) Suppose $\nu_{2}$ and $\nu_{3}$ are fixed so that conditions (E2) and (E3) of the equilibrium problem are satisfied. Then the measure $\nu_{1}$ that minimizes $E\left(\nu_{1}, \nu_{2}, \nu_{3}\right)$ subject to the condition (E1) exists. It has the following properties:

(i) If the constraint $\rho_{1}$ is not active, then the measure $\nu_{1}$ is given by

$$
\nu_{1}=\frac{1}{2} \operatorname{Bal}\left(\nu_{2}, \mathbb{R}^{-}\right),
$$

or, equivalently, in view of (3.3) and (3.5),

$$
\frac{d \nu_{1}(x)}{d x}=\frac{1}{2 \pi \sqrt{|x|}} \int \frac{\sqrt{s}}{s-x} d \nu_{2}(s), \quad x \in \mathbb{R}^{-} .
$$


(ii) If the constraint $\rho_{1}$ is active, then there exists a constant $r_{1}>0$, such that

$$
\operatorname{supp}\left(\rho_{1}-\nu_{1}\right)=\left(-\infty,-r_{1}\right] .
$$

Moreover, we have

$$
\begin{cases}2 U^{\nu_{1}}(x)=U^{\nu_{2}}(x), & x \in \operatorname{supp}\left(\rho_{1}-\nu_{1}\right), \\ 2 U^{\nu_{1}}(x)<U^{\nu_{2}}(x), & x \in \mathbb{R}^{-} \backslash \operatorname{supp}\left(\rho_{1}-\nu_{1}\right),\end{cases}
$$

and $\rho_{1}-\nu_{1}$ vanishes like a square root at $-r_{1}$.

(iii) Suppose $\nu_{2}$ has a compact support. Then here exists a constant $\tilde{K}>0$, depending only on $\max \operatorname{supp}\left(\nu_{2}\right)$ such that

$$
\frac{d \nu_{1}(x)}{d x} \leq \frac{\tilde{K}}{|x|^{3 / 2}}, \quad x \in \mathbb{R}^{-} .
$$

Proof. (i) Suppose that $\nu_{1}=\operatorname{Bal}\left(\nu_{2}, \mathbb{R}^{-}\right) / 2$. Then $2 U^{\nu_{1}}(x)=U^{\nu_{2}}(x)$ for $x \in \mathbb{R}^{-}$, which is the variational condition for (3.6) without constraint. This proves part (i).

(ii) Part (ii) is proved by using iterated balayage algorithm of [37]. The main idea is to construct a sequence of real numbers $\left\{r_{1, k}(t)\right\}$ and a sequence of measures $\left\{\nu_{1, k}\right\}$ with limit $r_{1}(t)$ and $\nu_{1}$, respectively. Since this argument is similar to those given in [23], we omit the proof here.

(iii) Suppose $\nu_{2}$ has a compact support and let

$$
b=\max \operatorname{supp}\left(\nu_{2}\right) .
$$

By part (ii), the constraint is active on some interval $\left[-r_{1}, 0\right)$ (which could be empty). Since $\left\|\nu_{1}\right\|=1 / 2$, we then have that $r_{1} \leq c_{1}$ where $c_{1}>0$ is the value for which $\rho_{1}\left(\left[-c_{1}, 0\right]\right)=1 / 2$. By properties of balayage we then have that the restriction of $\nu_{1}$ to $K_{c_{1}}$ is less than the balayage of $\nu_{2} / 2$ onto $K_{c_{1}}$. Hence by (3.3) and (3.4)

$$
\frac{d \nu_{1}(x)}{d x} \leq \frac{1}{2 \pi \sqrt{\left|x+c_{1}\right|}} \int \frac{\sqrt{s+c_{1}}}{s-x} d \nu_{2}(s), \quad x \in\left(-\infty,-c_{1}\right] .
$$

The function $s \mapsto \frac{\sqrt{s+c_{1}}}{s-x}$ is increasing for $s \in\left[-c_{1},-x-2 c_{1}\right]$. Thus if $x \leq-2 c_{1}-b$, then the maximum on $\operatorname{supp}\left(\nu_{2}\right)$ is taken at its right end point $s=b$, and therefore

$$
\frac{d \nu_{1}(x)}{d x} \leq \frac{1}{2 \pi \sqrt{\left|x+c_{1}\right|}} \frac{\sqrt{b+c_{1}}}{b-x}, \quad x \in\left(-\infty,-2 c_{1}-b\right] .
$$

Then there is a constant $\tilde{K}$, which only depends on $b$, such that (3.11) holds for $x \leq-2 c_{1}-b$. Since $\nu_{1} \leq \rho_{1}$, we can then adjust the constant if necessary, such that (3.11) holds for every $x<0$. 
If $\nu_{1}$ and $\nu_{2}$ are fixed, the equilibrium problem for $\nu_{3}$ is to minimize (3.6) among all measures $\nu$ on $\mathbb{R}^{-}$with total mass $1 / 2$ and satisfying the constraint $\nu \leq \rho_{3}$, where $\rho_{3}$ is given in (2.8). Hence, the properties of $\nu_{3}$ can be derived in a manner similar to the analysis given above. We omit the proof and state the results in the following lemma:

Lemma 3.2. (The measure $\nu_{3}$ ) Suppose $\nu_{1}$ and $\nu_{2}$ are fixed so that conditions (E1) and (E2) of the equilibrium problem are satisfied. Then the measure $\nu_{3}$ that minimizes $E\left(\nu_{1}, \nu_{2}, \nu_{3}\right)$ subject to the condition (E3) exists. It has the following properties:

(i) If the constraint $\rho_{3}$ is not active, the measure $\nu_{3}$ is given by

$$
\frac{d \nu_{3}(x)}{d x}=\frac{1}{2 \pi \sqrt{|x|}} \int \frac{\sqrt{z}}{z-x} d \nu_{2}(z), \quad x \in \mathbb{R}^{-} .
$$

(ii) If the constraint $\rho_{3}$ is active, there exists a constant $r_{3}(t)>0$, such that

$$
\operatorname{supp}\left(\rho_{3}-\nu_{3}\right)=\left(-\infty,-r_{3}(t)\right]
$$

Moreover, we have

$$
\begin{cases}2 U^{\nu_{3}}(x)=U^{\nu_{2}}(x), & x \in \operatorname{supp}\left(\rho_{3}-\nu_{3}\right), \\ 2 U^{\nu_{3}}(x)<U^{\nu_{2}}(x), & x \in \mathbb{R}^{-} \backslash \operatorname{supp}\left(\rho_{3}-\nu_{3}\right),\end{cases}
$$

and $\rho_{3}-\nu_{3}$ vanishes like a square root at $-r_{3}(t)$.

(iii) Suppose $\nu_{2}$ has a compact support. Then there exists a constant $\tilde{K}>0$, depending only on $\max \operatorname{supp}\left(\nu_{2}\right)$ such that

$$
\frac{d \nu_{3}(x)}{d x} \leq \frac{\tilde{K}}{|x|^{3 / 2}}, \quad x \in \mathbb{R}^{-} .
$$

\subsection{Equilibrium problem for $\nu_{2}$}

If $\nu_{1}$ and $\nu_{3}$ satisfying conditions $(E 1)$ and $(E 3)$ are fixed, the equilibrium problem for $\nu_{2}$ is to minimize

$$
I(\nu)+\int\left(V(x)-U^{\nu_{1}}(x)-U^{\nu_{3}}(x)\right) d \nu(x)
$$

among all probability measures $\nu$ on $\mathbb{R}^{+}$, where $V$ is given by (2.4). This is a usual equilibrium problem with external field

$$
V(x)-U^{\nu_{1}}(x)-U^{\nu_{3}}(x)
$$

see e.g. [15, 48]. 
Lemma 3.3. (The measure $\nu_{2}$ ) Suppose $\nu_{1}$ and $\nu_{3}$ are fixed so that conditions (E1) and (E3) in the equilibrium problem are satisfied, in particular we have $\nu_{j} \leq \rho_{j}$ for $j=1,3$. Then the measure $\nu_{2}$ that minimizes $E\left(\nu_{1}, \nu_{2}, \nu_{3}\right)$ subject to condition $(E 2)$ exists and has the following properties:

(i) The external field (3.19) is strictly convex on $\mathbb{R}^{+}$, and there exist two constants $0 \leq p<q$ such that

$$
\operatorname{supp}\left(\nu_{2}\right)=[p, q] .
$$

(ii) If $0 \notin \operatorname{supp}\left(\rho_{1}-\nu_{1}\right) \cup \operatorname{supp}\left(\rho_{3}-\nu_{3}\right)$ (so that both constraints are active in a neighborhood of 0 ), then (3.19) is real analytic on $\mathbb{R}^{+}$(including 0 ).

Proof. (i) The second derivative of (3.19) is, for $x>0$,

$$
\begin{aligned}
& V^{\prime \prime}(x)-\left(U^{\nu_{1}}\right)^{\prime \prime}(x)-\left(U^{\nu_{3}}\right)^{\prime \prime}(x) \\
& =\frac{\sqrt{a}}{2 t} x^{-3 / 2}+\frac{\sqrt{b}}{2(1-t)} x^{-3 / 2}-\int_{-\infty}^{0} \frac{d \nu_{1}(s)}{(x-s)^{2}}-\int_{-\infty}^{0} \frac{d \nu_{3}(s)}{(x-s)^{2}} .
\end{aligned}
$$

Since $\nu_{1}$ satisfies the constraint $\nu_{1} \leq \rho_{1}$ we have for $x>0$,

$$
\int_{-\infty}^{0} \frac{d \nu_{1}(s)}{(x-s)^{2}}<\int_{-\infty}^{0} \frac{d \rho_{1}(s)}{(x-s)^{2}}=\frac{\sqrt{a}}{\pi t} \int_{-\infty}^{0} \frac{d s}{\sqrt{|s|}(x-s)^{2}}=\frac{\sqrt{a}}{2 t} x^{-3 / 2}
$$

where we used the explicit expression (2.6) for $\rho_{1}$. The above inequality is strict since $\nu_{1}$ cannot be equal to $\rho_{1}$ on the whole negative real axis. Similarly, we find

$$
\int_{-\infty}^{0} \frac{d \nu_{3}(s)}{(x-s)^{2}}<\frac{\sqrt{b}}{2(1-t)} x^{-3 / 2}, \quad x>0 .
$$

Combining (3.21)-(3.23), we find that (3.21) is positive, and therefore (3.19) is strictly convex. It then follows from [48, Theorem IV.1.10] that the support of $\nu_{2}$ consists of one interval, say, $[p, q]$ with $0 \leq p<q$.

(ii) Suppose that $s>0$ is such that $\nu_{1}=\rho_{1}$ and $\nu_{3}=\rho_{3}$ on $(-s, 0)$. Thus, if $x \geq 0$,

$$
-U^{\nu_{1}}(x)=\int_{-s}^{0} \log |x-y| d \rho_{1}(y)+\int_{-\infty}^{-s} \log |x-y| d \rho_{1}(y)
$$

where the second term is real analytic on $\mathbb{R}^{+}$, and for the first term we use the explicit expression (2.6) for $\rho_{1}$. Then we obtain for the derivative of (3.24) (where "real analytic" means a real analytic function on $\mathbb{R}^{+}$)

$$
\begin{aligned}
\left(-U^{\nu_{1}}\right)^{\prime}(x) & =\frac{\sqrt{a}}{\pi t} \int_{-s}^{0} \frac{d y}{(x-y) \sqrt{-y}}+\text { "real analytic" } \\
& =\frac{\sqrt{a}}{\pi t} \int_{-\infty}^{0} \frac{d y}{(x-y) \sqrt{-y}}+\text { "real analytic" } \\
& =\frac{\sqrt{a}}{t} x^{-1 / 2}+\text { "real analytic". }
\end{aligned}
$$


Then by integrating (3.25) we find that $U^{\nu_{1}}(x)+\frac{2 \sqrt{a x}}{t}$ is real analytic for $x \in \mathbb{R}^{+}$ (including at $x=0$ ).

Similarly, we find that $U^{\nu_{3}}(x)+\frac{2 \sqrt{b x}}{1-t}$ is real analytic on $\mathbb{R}^{+}$as well. Then $V$ is real analytic because of (2.4) and (3.19).

\subsection{Proofs of Theorem 2.1 and Proposition 2.2}

Proof of uniqueness. We rewrite the energy functional $E\left(\nu_{1}, \nu_{2}, \nu_{3}\right)$ as

$$
E\left(\nu_{1}, \nu_{2}, \nu_{3}\right)=\frac{1}{2} I\left(\nu_{2}\right)+\frac{1}{4} I\left(\nu_{2}-2 \nu_{1}\right)+\frac{1}{4} I\left(\nu_{2}-2 \nu_{3}\right)+\int V(x) d \nu_{2}(x) .
$$

It is well-known that if two distinct measures $\nu$ and $\mu$ have finite logarithmic energy and $\int d \nu=\int d \mu$, then

$$
I(\nu-\mu) \geq 0 .
$$

Hence, it follows that the energy functional is strictly convex. The minimizer is therefore uniquely determined by the Euler-Lagrange variational conditions listed in Proposition 2.2.

Proof of existence. It is easily seen from (3.26)-(3.27) that

$$
E\left(\nu_{1}, \nu_{2}, \nu_{3}\right) \geq \frac{1}{2} I\left(\nu_{2}\right)+\int V(x) d \nu_{2}(x)
$$

Thus the energy functional is bounded from below. As a consequence, we can find a sequence $\left(\nu_{1, n}, \nu_{2, n}, \nu_{3, n}\right)$ such that

$$
E\left(\nu_{1, n}, \nu_{2, n}, \nu_{3, n}\right) \leq \frac{1}{n}+\inf E\left(\nu_{1}, \nu_{2}, \nu_{3}\right),
$$

where both the sequence and the infimum are taken subject to conditions $(E 1)-(E 3)$ of the equilibrium problem. In addition, we may assume that

$$
\operatorname{supp}\left(\nu_{2, n}\right) \subset[p, q],
$$

and, in view of $(3.11),(3.17)$,

$$
\frac{d \nu_{i, n}(x)}{d x} \leq \frac{\tilde{K}}{|x|^{3 / 2}}, \quad i=1,3
$$

with $0 \leq p<q$ and $\tilde{K}>0$ independent of $n$. Therefore, there exists a weakly convergent subsequence of $\left(\nu_{1, n}, \nu_{2, n}, \nu_{3, n}\right)$, with a certain weak limit $\left(\nu_{1}, \nu_{2}, \nu_{3}\right)$. By the fact that the energy functional $E$ is lower semi-continuous [29], the vector of measures $\left(\nu_{1}, \nu_{2}, \nu_{3}\right)$ is then the required minimizer of the energy minimization problem. 
Proofs of Theorem 2.1 and Proposition 2.2. We already showed that there exists a unique minimizer $\left(\mu_{1}, \mu_{2}, \mu_{3}\right)$ for the equilibrium problem. The parts (a)-(c) of Theorem 2.1 and Proposition 2.2 are now immediate by Lemmas 3.1-3.3.

\section{Riemann surface and auxiliary functions}

In this section, we shall construct a Riemann surface $\mathcal{R}$ with the minimizer $\left(\mu_{1}, \mu_{2}, \mu_{3}\right)$ of the vector equilibrium problem and introduce some auxiliary functions for further use.

\subsection{A four-sheeted Riemann surface $\mathcal{R}$}

The Riemann surface $\mathcal{R}$ has four sheets $\mathcal{R}_{j}, j=1, \ldots, 4$, which are defined as

$$
\begin{aligned}
& \mathcal{R}_{1}=\mathbb{C} \backslash \operatorname{supp}\left(\rho_{1}-\mu_{1}\right), \quad \mathcal{R}_{2}=\mathbb{C} \backslash\left(\operatorname{supp}\left(\rho_{1}-\mu_{1}\right) \cup \operatorname{supp}\left(\mu_{2}\right)\right), \\
& \mathcal{R}_{3}=\mathbb{C} \backslash\left(\operatorname{supp}\left(\mu_{2}\right) \cup \operatorname{supp}\left(\rho_{3}-\mu_{3}\right)\right), \quad \mathcal{R}_{4}=\mathbb{C} \backslash \operatorname{supp}\left(\rho_{3}-\mu_{3}\right) .
\end{aligned}
$$

These four sheets are connected as follows: $\mathcal{R}_{1}$ is connected to $\mathcal{R}_{2}$ via $\operatorname{supp}\left(\rho_{1}-\mu_{1}\right), \mathcal{R}_{2}$ is connected to $\mathcal{R}_{3}$ via $\operatorname{supp}\left(\mu_{2}\right)$, and $\mathcal{R}_{3}$ is connected to $\mathcal{R}_{4}$ via $\operatorname{supp}\left(\rho_{3}-\mu_{3}\right)$. Each connection is in the usual crosswise manner; see Figure 3.

The Riemann surface is compactified by adding two points at infinity: one is common to the sheets $\mathcal{R}_{1}$ and $\mathcal{R}_{2}$, and the other one is common to the sheets $\mathcal{R}_{3}$ and $\mathcal{R}_{4}$. Since the Riemann surface $\mathcal{R}$ has 4 sheets and 6 simple branch points, it then follows from Hurwitz's theorem (see e.g. [45]) that it has genus 0. This fact will be helpful in our future construction of the global parametrix.

\subsection{The $\xi$-functions}

We use $F_{j}$ to denote the Cauchy transform of the measure $\mu_{j}$, i.e.,

$$
F_{j}(z)=\int \frac{1}{z-x} d \mu_{j}(x), \quad z \in \mathbb{C} \backslash \operatorname{supp}\left(\mu_{j}\right), \quad j=1,2,3 .
$$

With the above notation, the Sokhotski-Plemelj formulas now read

$$
\begin{aligned}
& F_{j,+}(z)+F_{j,-}(z)=2 \mathrm{PV} \int \frac{1}{z-x} d \mu_{j}(x), \\
& F_{j,+}(z)-F_{j,-}(z)=-2 \pi i \frac{d \mu_{j}}{d z},
\end{aligned}
$$

for $z \in \operatorname{supp}\left(\mu_{j}\right)$, where PV denotes the Cauchy principal value. The $\xi$-functions are then defined as follows.

Theorem 4.1. The function $\xi: \bigcup_{j=1}^{4} \mathcal{R}_{j} \rightarrow \mathbb{C}$ defined by

$$
\xi(z)= \begin{cases}-F_{1}(z)+\frac{\sqrt{a}}{t \sqrt{z}}+\frac{1}{t(1-t)}, & z \in \mathcal{R}_{1}, \\ F_{1}(z)-F_{2}(z)-\frac{\sqrt{a}}{t \sqrt{z}}+\frac{1}{t(1-t)}, & z \in \mathcal{R}_{2}, \\ F_{2}(z)-F_{3}(z)+\frac{\sqrt{b}}{(1-t) \sqrt{z}}, & z \in \mathcal{R}_{3}, \\ F_{3}(z)-\frac{\sqrt{b}}{(1-t) \sqrt{z}}, & z \in \mathcal{R}_{4},\end{cases}
$$



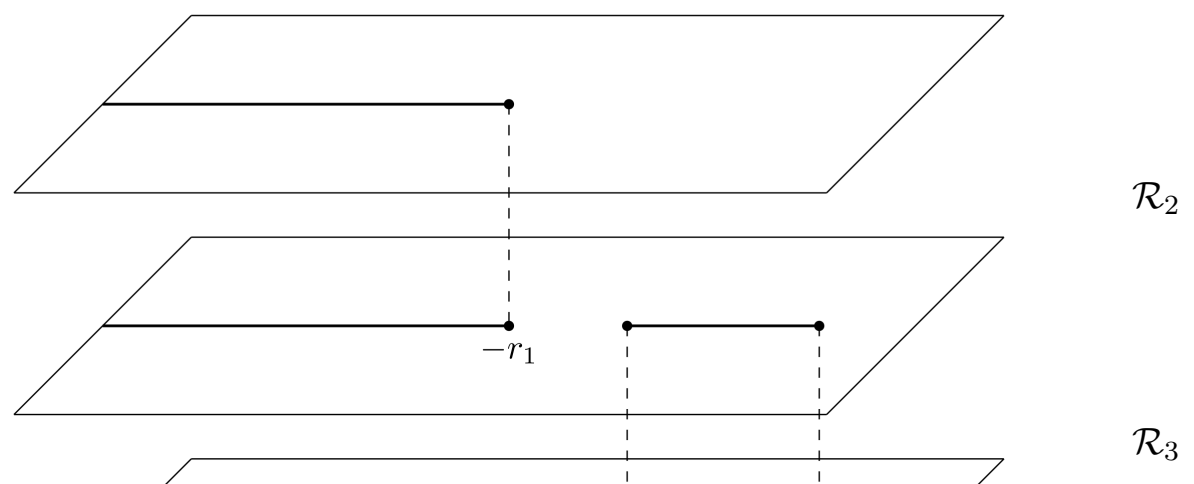

$\mathcal{R}_{3}$

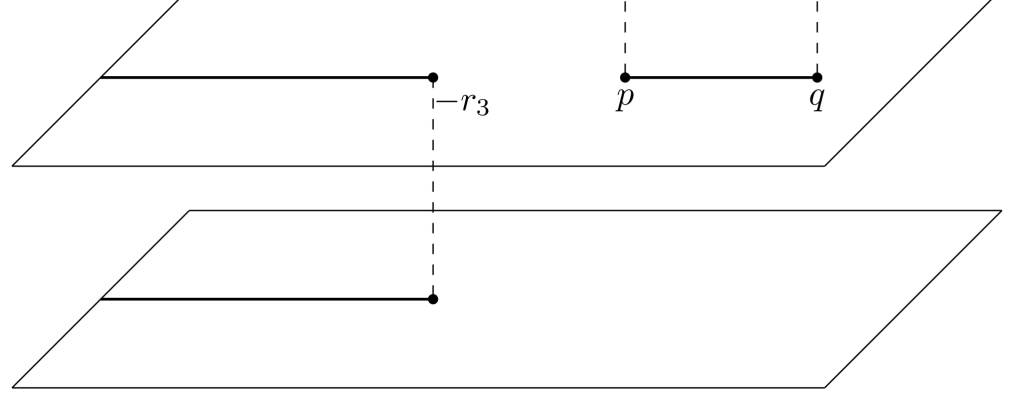

$\mathcal{R}_{4}$

Figure 3: The Riemann surface $\mathcal{R}$.

has an extension to a meromorphic function (also denoted by $\xi$ ) on $\mathcal{R}$. In (4.5), the principal branch of the square root function $\sqrt{z}$ is used, i.e., with a branch cut along $(-\infty, 0]$.

Proof. We first need to show the analyticity of $\xi$ on each individual sheet $\mathcal{R}_{j}$. To see this, we note that $\mu_{1}=\rho_{1}$ on $\mathbb{R}^{-} \backslash \operatorname{supp}\left(\rho_{1}-\mu_{1}\right)=\left(-r_{1}, 0\right)$. It then follows from (4.4) and the definition (2.6) of $\rho_{1}$ that

$$
\begin{aligned}
F_{1,+}(x)-F_{1,-}(x) & =-2 \pi i \frac{d \mu_{1}(x)}{d x}=-2 \pi i \frac{d \rho_{1}(x)}{d x} \\
& =-2 i \frac{\sqrt{a}}{t|x|^{1 / 2}}=\frac{\sqrt{a}}{t}\left(\frac{1}{\sqrt{x}_{+}}-\frac{1}{\sqrt{x_{-}}}\right),
\end{aligned}
$$

for $x \in\left(-r_{1}, 0\right)$. By the definition of $\xi$, this implies that $\xi$ has an analytic continuation to $\left(-r_{1}, 0\right)$ on both $\mathcal{R}_{1}$ and $\mathcal{R}_{2}$. Similarly, we have by (4.4) and (2.8)

$$
F_{3,+}(x)-F_{3,-}(x)=\frac{\sqrt{b}}{1-t}\left(\frac{1}{\sqrt{x}_{+}}-\frac{1}{\sqrt{x}_{-}}\right),
$$


for $x \in\left(-r_{2}, 0\right)$, which gives the analyticity of $\xi$ on the sheets $\mathcal{R}_{3}$ and $\mathcal{R}_{4}$.

It remains to check the analyticity of $\xi$ if one moves from one sheet to another via a specific cut. This is always the case in view of the variational conditions for the equilibrium problem. For convenience, we will only show that $\xi$ is analytic when one crosses $\operatorname{supp}\left(\rho_{1}-\mu_{1}\right)$, since the other cases can be verified similarly.

We start with differentiating $U^{\mu_{1}}$ on $\operatorname{supp}\left(\rho_{1}-\mu_{1}\right)$ and obtain

$$
2 \frac{d}{d x} U^{\mu_{1}}(x)=-2 \mathrm{PV} \int \frac{1}{x-s} d \mu_{1}(s)=-F_{1,+}(x)-F_{1,-}(x),
$$

where the second equality follows from Sokhotski-Plemelj formula (4.3). On the other hand, differentiating the right-hand side of the variational condition (2.17), we have

$$
2 \frac{d}{d x} U^{\mu_{1}}(x)=\frac{d}{d x} U^{\mu_{2}}(x)=-\int \frac{1}{x-s} d \mu_{2}(s)=-F_{2}(x) .
$$

Combining (4.8) and (4.9) leads to

$$
F_{1,+}(x)+F_{1,-}(x)=F_{2}(x), \quad x \in \operatorname{supp}\left(\rho_{1}-\mu_{1}\right) .
$$

This, together with the fact that $\sqrt{x}_{-}=-\sqrt{x}_{+}$for $x \in \mathbb{R}^{-}$, gives the analyticity of $\xi$ when one crosses $\operatorname{supp}\left(\rho_{1}-\mu_{1}\right)$ and moves from $\mathcal{R}_{1}$ to $\mathcal{R}_{2}$.

As a consequence of the above theorem, the following result about the asymptotic behavior of $F_{j}(z)$ at infinity is easy to prove.

Lemma 4.2. There exist two constants $c_{1}$ and $c_{3}$ such that

$$
\begin{aligned}
& F_{1}(z)=\frac{1}{2 z}-\frac{c_{1}}{z^{3 / 2}}+O\left(\frac{1}{z^{2}}\right), \\
& F_{2}(z)=\frac{1}{z}+O\left(\frac{1}{z^{2}}\right) \\
& F_{3}(z)=\frac{1}{2 z}-\frac{c_{3}}{z^{3 / 2}}+O\left(\frac{1}{z^{2}}\right),
\end{aligned}
$$

as $z \rightarrow \infty$.

We use $\xi_{j}(z)$ to denote the restriction of $\xi$ to $\mathcal{R}_{j}$. The relations between $\xi_{j}$ and the solution to the equilibrium problem can be established in the following way.

Proposition 4.3. We have that

$$
\begin{aligned}
d \mu_{1}(x) & =\frac{1}{2 \pi i}\left(\xi_{1,+}(x)-\xi_{1,-}(x)\right) d x+d \rho_{1}(x), & & x \in \mathbb{R}^{-}, \\
d \mu_{2}(x) & =\frac{1}{2 \pi i}\left(\xi_{2,+}(x)-\xi_{2,-}(x)\right) d x, & & x \in \mathbb{R}^{+}, \\
d \mu_{3}(x) & =\frac{1}{2 \pi i}\left(\xi_{3,+}(x)-\xi_{3,-}(x)\right) d x+d \rho_{3}(x), & & x \in \mathbb{R}^{-} .
\end{aligned}
$$


Proof. This follows by a combination of (4.5), the Sokhotski-Plemelj formula (4.4), and the definitions (2.6) and (2.8) of $\rho_{1}$ and $\rho_{2}$.

The Riemann surface has two points at infinity and the meromorphic function (4.5) takes on two distinct finite values at these points (namely $\frac{1}{t(1-t)}$ and 0 ). Thus $\xi$ is not a constant, and being a meromorphic function on a compact Riemann surface it must have at least one pole. From (4.5) it is clear that the only possible poles are at the values of $z=0$ that correspond to branch points. It thus follows that at least one of $r_{1}, p$, and $r_{3}$ is zero, and we can identify the various cases in terms of the structure of $\mathcal{R}$.

In Case I we have $r_{1}=r_{3}=0$ and $p>0$. It is only in this case that $\xi$ has two poles. In all other cases, $\xi$ has exactly one pole, and then $\xi$ is actually a conformal map from $\mathcal{R}$ onto the Riemann sphere.

\subsection{The spectral curve}

Next we calculate the spectral curve associated to the vector equilibrium problem; see also [5] and the references therein for similar calculations. From Theorem 4.1, it follows that the four function $\xi_{j}(z), j=1,2,3,4$ are solutions to an algebraic equation of degree four

$$
\begin{aligned}
0=P(\xi, z)=\left(\xi-\xi_{1}\right)\left(\xi-\xi_{2}\right)\left(\xi-\xi_{3}\right) & \left(\xi-\xi_{4}\right) \\
= & \xi^{4}+A(z) \xi^{3}+B(z) \xi^{2}+C(z) \xi+D(z),
\end{aligned}
$$

with coefficients $A(z), B(z), C(z), D(z)$ that can only have a pole in $z=0$. In fact, because of the behavior of (4.5) at $z=0$, we have that $A(z)$ has no pole at $z=0$ (and therefore is a constant), $B(z)$ and $C(z)$ have at most a simple pole at $z=0$. Also $D(z)$ has at most a simple pole at $z=0$, unless we are in Case I, in which case $D(z)$ has a double pole at $z=0$. Indeed, in this last case we have that all functions $\xi_{j}$ behave like $z^{-1 / 2}$ as $z \rightarrow 0$, and then $D(z)=\xi_{1}(z) \xi_{2}(z) \xi_{3}(z) \xi_{4}(z)$ behaves like $z^{-2}$ as $z \rightarrow 0$.

Straightforward calculations using (4.5) and Lemma 4.2 yield in all cases

$$
\begin{aligned}
A(z) & =-\xi_{1}-\xi_{2}-\xi_{3}-\xi_{4}=-\frac{2}{t(1-t)} \\
B(z) & =\xi_{1} \xi_{2}+\xi_{1} \xi_{3}+\xi_{1} \xi_{4}+\xi_{2} \xi_{3}+\xi_{2} \xi_{4}+\xi_{3} \xi_{4} \\
& =\frac{1}{t^{2}(1-t)^{2}}-\frac{b}{z(1-t)^{2}}-\frac{a}{z t^{2}}+\frac{1}{z t(1-t)}, \\
C(z) & =\frac{2 b}{z t(1-t)^{3}}-\frac{1}{z t^{2}(1-t)^{2}}, \\
D(z) & =-\frac{b}{z t^{2}(1-t)^{4}}+\frac{c}{z^{2} t^{2}(1-t)^{2}},
\end{aligned}
$$

where $c=0$ in all cases but in Case I. 
Lemma 4.4. The spectral curve is given by (4.17) with coefficients given by (4.18)(4.21). The constant $c$ that appears in (4.21) is equal to

$$
c= \begin{cases}\left(\sqrt{a b}-\frac{1}{2}\right)^{2} & \text { in Case I, } \\ 0 & \text { in all other cases }\end{cases}
$$

In Case I, we have

$$
\begin{aligned}
& \sqrt{p}=(1-t) \sqrt{a}+t \sqrt{b}-\sqrt{2 t(1-t)}>0, \\
& \sqrt{q}=(1-t) \sqrt{a}+t \sqrt{b}+\sqrt{2 t(1-t)} .
\end{aligned}
$$

Proof. We already saw that the spectral curve is given by (4.17), (4.18)-(4.21) and that $c=0$ in all cases, except in Case I.

Now suppose we are in Case I, so that $r_{1}=r_{3}=0$ and $p>0$. Consider the following functions

$$
\begin{aligned}
& \eta_{1}(z)=\left\{\begin{array}{ll}
z \xi_{2}\left(z^{2}\right), & \operatorname{Re} z>0, \\
z \xi_{1}\left(z^{2}\right), & \operatorname{Re} z<0,
\end{array} \quad \eta_{2}(z)= \begin{cases}z \xi_{1}\left(z^{2}\right), & \operatorname{Re} z>0, \\
z \xi_{2}\left(z^{2}\right), & \operatorname{Re} z<0,\end{cases} \right. \\
& \eta_{3}(z)=\left\{\begin{array}{ll}
z \xi_{3}\left(z^{2}\right), & \operatorname{Re} z>0, \\
z \xi_{4}\left(z^{2}\right), & \operatorname{Re} z<0,
\end{array} \quad \eta_{4}(z)= \begin{cases}z \xi_{4}\left(z^{2}\right), & \operatorname{Re} z>0, \\
z \xi_{3}\left(z^{2}\right), & \operatorname{Re} z<0\end{cases} \right.
\end{aligned}
$$

Since, we are in Case I, we have that all functions $\eta_{j}$ are analytic across the imaginary axis. Also the singularities at $z=0$ are removable because of the extra factors $z$ in (4.24) and (4.25). It follows that $\eta_{1}$ and $\eta_{3}$ are analytic in $\mathbb{C} \backslash[\sqrt{p}, \sqrt{q}]$, while $\eta_{2}$ and $\eta_{4}$ are analytic in $\mathbb{C} \backslash[-\sqrt{q},-\sqrt{p}]$.

In fact the two functions $\eta_{1}$ and $\eta_{3}$ give a meromorphic function on the two-sheeted Riemann surface with cut along $[\sqrt{p}, \sqrt{q}]$, whose only pole is at infinity on the first sheet. Since

$$
\begin{aligned}
& \eta_{1}(z)=\frac{z}{t(1-t)}-\frac{\sqrt{a}}{t}-\frac{1}{2 z}+O\left(z^{-2}\right) \\
& \eta_{3}(z)=\frac{\sqrt{b}}{1-t}+\frac{1}{2 z}+O\left(z^{-2}\right)
\end{aligned}
$$

as $z \rightarrow \infty$, it then follows that $\eta_{1}(z)$ and $\eta_{3}(z)$ are the two solutions of the quadratic equation

$$
\eta^{2}-\left(\frac{z}{t(1-t}-\frac{\sqrt{a}}{t}+\frac{\sqrt{b}}{1-t}\right) \eta+\left(\frac{\sqrt{b} z}{t(1-t)^{2}}-\frac{\sqrt{a b}}{t(1-t)}+\frac{1}{2 t(1-t)}\right)=0 .
$$

Similarly, $\eta_{2}(z)$ and $\eta_{4}(z)$ are the two solutions of

$$
\eta^{2}-\left(\frac{z}{t(1-t)}+\frac{\sqrt{a}}{t}-\frac{\sqrt{b}}{1-t}\right) \eta+\left(-\frac{\sqrt{b} z}{t(1-t)^{2}}-\frac{\sqrt{a b}}{t(1-t)}+\frac{1}{2 t(1-t)}\right)=0 .
$$


In particular, it follows that

$$
\eta_{1}(z) \eta_{2}(z) \eta_{3}(z) \eta_{4}(z)=-\frac{b z^{2}}{t^{2}(1-t)^{4}}+\frac{\left(\sqrt{a b}-\frac{1}{2}\right)^{2}}{t^{2}(1-t)^{2}}
$$

Then in view of (4.24) and (4.25) we obtain

$$
z^{4} D\left(z^{2}\right)=z^{4} \xi_{1}\left(z^{2}\right) \xi_{2}\left(z^{2}\right) \xi_{3}\left(z^{2}\right) \xi_{4}\left(z^{2}\right)=-\frac{b z^{2}}{t^{2}(1-t)^{4}}+\frac{\left(\sqrt{a b}-\frac{1}{2}\right)^{2}}{t^{2}(1-t)^{2}} .
$$

Comparing this with (4.21) we indeed find that $c$ is given by (4.22) in Case I.

The branch points of (4.26) are equal to $\sqrt{p}$ and $\sqrt{q}$. Simple calculations then lead to $(4.23)$.

Remark 1. We notice that the spectral curve in Case I is equivalent with the one in [18], while in Cases II and III it is equivalent with the one in [14] (with $\sqrt{a}, \sqrt{b}$ instead of $a, b)$. This implies that the limiting distribution of the non-intersecting squared Bessel paths is obtained from the one of the corresponding non-intersecting Brownian motions by a simple squaring transform.

One could extend the above ideas even further by also lifting the vector equilibrium problem into its square-root form. The lifted measures are then supported on the real or imaginary axis. This approach can be used to obtain an equilibrium problem interpretation for the spectral curve in [14], which allows in turn to obtain an alternative derivation of the results in that paper. We will not go into the details.

Proposition 4.5. We are in Case I if and only if $a b>1 / 4$.

Proof. The spectral curve depends continuously on the parameters $a, b$ and $t$. Therefore the change from Case I to one of the other cases can only occur when $a b=1 / 4$.

If we are in Case I, then $\sqrt{p}$ is given by (4.23) which in particular implies that $(1-t) \sqrt{a}+t \sqrt{b}-\sqrt{2 t(1-t)}>0$. For $t=1 / 2$ and $a=b<1 / 2$ this inequality is not satisfied and so for these values of parameters we are not in Case I. Then it follows that for all $a$ and $b$ with $a b \leq 1 / 4$ we are not in Case I.

If we are not in Case I, then $c=0$ and the spectral curve (4.17) has four real branch points $-r_{1},-r_{2}, p$ and $q$. For the specific values $t=1 / 2, a=1$ and $b=1 / 2$, we calculated with Maple that there are two non-real branch points. Thus for those values of parameters we cannot have $c=0$, and so are in Case I. By continuity we are in Case I for all $a$ and $b$ with $a b>1 / 4$. This completes the proof of the proposition.

\subsection{Phase transitions}

Let us assume that the ending point $b$ is fixed. In figure 4 we give a phase diagram divided into three different regions according with the limiting distribution of the nonintersecting squared Bessel paths as $n \rightarrow \infty$. The horizontal axis of the figure denotes the time $t \in(0,1)$ and the vertical axis denotes the starting point $a$. 

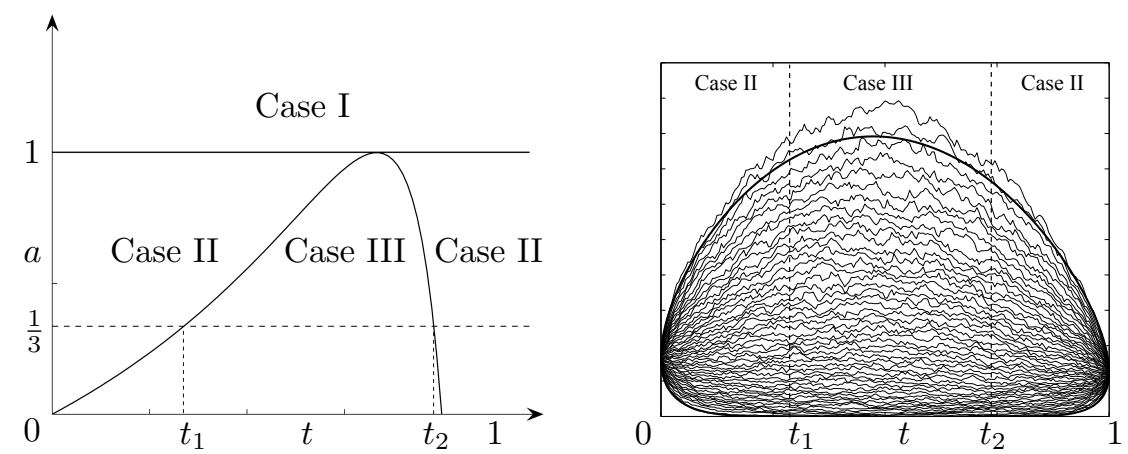

Figure 4: The left figure shows the phase diagram for the non-intersecting squared Bessel paths for $b=1 / 4$. The solid curve is given in (4.27). The right figure shows a simulation of 50 non-intersecting paths for $a=1 / 3$ which corresponds to the dashed line in the left figure.

In the case $a b<1 / 4$, for $t$ small, we are in Case II. The paths start at the positive value $a$ at $t=0$ and they initially remain positive. The limiting mean density is supported in the interval $[p, q]$ with $p>0$ (see Theorems 2.1 and 2.3). For this case there is only one active constraint.

We have computed the discriminant $\operatorname{Disc}(z)$ of (4.17) with coefficients given by (4.18)-(4.21) and $c=0$ in (4.21) with respect to $\xi$ using Maple and we obtain

$$
\operatorname{Disc}(z)=a_{4} z^{4}+a_{3} z^{3}+a_{2} z^{2}+4(4 a b-1)\left(a-2 a t+a t^{2}+b t^{2}+t^{2}-t\right)^{3} z
$$

for some constants $a_{4}, a_{3}$ and $a_{2}$. We do not include the explicit expressions of $a_{4}, a_{3}$ and $a_{2}$ because they are too long and not too interesting. Therefore there are exactly four zeros $\operatorname{Disc}(z)$ which correspond to the branch points of the Riemann surface.

For each $a b<1 / 4$ there are two critical times $t_{1,2}$ where $\operatorname{Disc}(z)$ has a double zero at $z=0$, namely

$$
t_{1,2}=\frac{2 a+1 \pm \sqrt{1-4 a b}}{2(a+b+1)} .
$$

The critical times $t_{1}$ and $t_{2}$ are the only two values of $t$ for which the coefficient of $z$ in $\operatorname{Disc}(z)$ is equal to zero. The time $t_{1}$ corresponds to the time where the first paths touch the wall. If $t_{1}<t<t_{2}$ then we are in Case III. Here the limiting mean density of the paths is supported on the interval $[0, q]$ (see Theorems 2.1 and 2.3). Both constraints are active in this case. If $t>t_{2}$ then we are back to the Case II.

The figure on the right of Figure 4 shows the transitions between Case II and Case III for the choice of the parameters $a=1 / 3$ and $b=1 / 4$. This choice corresponds to the dashed line in the left figure. The critical times $t_{1}$ and $t_{2}$ where the transition occurs are given by (4.27).

On the other hand, if $a b>1 / 4$ then the paths start at the positive value $a$ at $t=0$ and they remain positive. In this case the limiting mean density is supported in the interval $[p, q]$ with $p>0$. No constraint is active in this case. 


\subsection{The $\lambda$-functions}

For the measures $\mu_{j}, j=1,2,3$ that minimize the energy functional (2.3), we first define the associated $g$-functions as

$$
g_{j}(z)=\int \log (z-s) d \mu_{j}(s)
$$

where we take the principal branch of the logarithm, i.e.,

$$
\log (z-s)=\log |z-s|+i \arg (z-s), \quad \arg (z-s) \in(-\pi, \pi) .
$$

On account of Theorem 2.1 , it is easily seen that $g_{1}$ and $g_{3}$ are analytic in $\mathbb{C} \backslash \mathbb{R}^{-}$, while $g_{2}$ is analytic in $\mathbb{C} \backslash(-\infty, q]$.

Noting that

$$
g_{j}^{\prime}(z)=F_{j}(z),
$$

we find the asymptotic behavior of $g_{j}(z)$ at infinity as given in the following lemma.

Lemma 4.6. With the constants $c_{1}$ and $c_{3}$ given in Lemma 4.2, we have that

$$
\begin{aligned}
& g_{1}(z)=\frac{1}{2} \log z+\frac{2 c_{1}}{\sqrt{z}}+O\left(\frac{1}{z}\right), \\
& g_{2}(z)=\log z+O\left(\frac{1}{z}\right) \\
& g_{3}(z)=\frac{1}{2} \log z+\frac{2 c_{3}}{\sqrt{z}}+O\left(\frac{1}{z}\right),
\end{aligned}
$$

as $z \rightarrow \infty$.

Proof. The asymptotics of $g_{2}$ follows from the fact that $\mu_{2}$ is a probability measure with compact support.

The formulas (4.31) and (4.33) for $g_{1}$ and $g_{3}$ can be derived by integrating the asymptotic formulas for $F_{1}$ and $F_{3}$ given in Lemma 4.2. The integration constant is zero, due to the fact that

$$
\int \log (z-s) d \mu_{j}(s)=\log (z)\left\|\mu_{j}\right\|+\int \log \left(1-\frac{s}{z}\right) d \mu_{j}(s), \quad j=1,3,
$$

and $\int \log (1-s / z) d \mu_{j}(s) \rightarrow 0$ as $z \rightarrow \infty$.

The $\lambda$-functions are then defined as the following anti-derivatives of the $\xi$-functions:

$$
\begin{aligned}
& \lambda_{1}(z)=-g_{1}(z)+\frac{2 \sqrt{a z}}{t}+\frac{z}{t(1-t)}-l, \\
& \lambda_{2}(z)=g_{1}(z)-g_{2}(z)-\frac{2 \sqrt{a z}}{t}+\frac{z}{t(1-t)}-l, \\
& \lambda_{3}(z)=g_{2}(z)-g_{3}(z)+\frac{2 \sqrt{b z}}{1-t}, \\
& \lambda_{4}(z)=g_{3}(z)-\frac{2 \sqrt{b z}}{1-t}
\end{aligned}
$$


where $l \in \mathbb{R}$ is the variational constant in Proposition 2.2. Note that $\lambda_{1}$ and $\lambda_{4}$ are analytic in $\mathbb{C} \backslash(-\infty, 0]$ while $\lambda_{2}$ and $\lambda_{3}$ are analytic in $\mathbb{C} \backslash(-\infty, q]$. Some properties of $\lambda$-functions are shown in the following two lemmas.

Lemma 4.7. We have

$$
\begin{array}{ll}
\lambda_{1,+}(x)=\lambda_{1,-}(x), & x \in\left(-r_{1}, 0\right), \\
\lambda_{2,+}(x)=\lambda_{2,-}(x)-2 \pi i, & x \in\left(-r_{1}, p\right), \\
\lambda_{3,+}(x)=\lambda_{3,-}(x)+2 \pi i, & x \in\left(-r_{3}, p\right), \\
\lambda_{4,+}(x)=\lambda_{4,-}(x), & x \in\left(-r_{3}, 0\right), \\
\lambda_{1, \pm}(x)=\lambda_{2, \mp}(x) \mp \pi i, & x \in \Delta_{1}, \\
\lambda_{2, \pm}(x)=\lambda_{3, \mp}(x), & x \in \Delta_{2}, \\
\lambda_{3, \pm}(x)=\lambda_{4, \mp}(x) \mp \pi i, & x \in \Delta_{3},
\end{array}
$$

and

$$
\begin{array}{ll}
\operatorname{Re}\left(\lambda_{1}(x)-\lambda_{2}(x)\right)<0, & x \in\left(-r_{1}, 0\right), \\
\operatorname{Re}\left(\lambda_{2}(x)-\lambda_{3}(x)\right)>0, & x \in \mathbb{R}^{+} \backslash \Delta_{2}, \\
\operatorname{Re}\left(\lambda_{3}(x)-\lambda_{4}(x)\right)<0, & x \in\left(-r_{3}, 0\right) .
\end{array}
$$

Proof. These are reformulations of the variational conditions of the equilibrium problem in Proposition 2.2.

Lemma 4.8. The $\lambda$-functions have the following asymptotics for $z \rightarrow \infty$ :

$$
\begin{aligned}
& \lambda_{1}(z)=\frac{z}{t(1-t)}+\frac{2 \sqrt{a z}}{t}-l-\frac{1}{2} \log z-\frac{2 c_{1}}{\sqrt{z}}+O\left(z^{-1}\right), \\
& \lambda_{2}(z)=\frac{z}{t(1-t)}-\frac{2 \sqrt{a z}}{t}-l-\frac{1}{2} \log z+\frac{2 c_{1}}{\sqrt{z}}+O\left(z^{-1}\right), \\
& \lambda_{3}(z)=\frac{2 \sqrt{b z}}{1-t}+\frac{1}{2} \log z-\frac{2 c_{3}}{\sqrt{z}}+O\left(z^{-1}\right), \\
& \lambda_{4}(z)=-\frac{2 \sqrt{b z}}{1-t}+\frac{2 c_{3}}{\sqrt{z}}+\frac{1}{2} \log z+O\left(z^{-1}\right) .
\end{aligned}
$$

Proof. Obvious from the definitions (4.34)-(4.37) and from (4.31)-(4.33).

\subsection{The $\phi$-functions}

We end this section with the introduction of the $\phi$-functions, which will be used to simplify the jump matrices during our steepest descent analysis. Recall that $\xi_{j}$ is the 
restriction of $\xi$ to the sheet $\mathcal{R}_{j}$ of the Riemann surface $\mathcal{R}$. We define

$$
\begin{aligned}
& \phi_{1}(z)=\frac{1}{2} \int_{-r_{1}}^{z}\left(\xi_{1}(y)-\xi_{2}(y)\right) d y, \quad z \in \mathbb{C} \backslash\left(\left(-\infty,-r_{1}\right] \cup[p, \infty)\right), \\
& \phi_{2}(z)=\frac{1}{2} \int_{q}^{z}\left(\xi_{2}(y)-\xi_{3}(y)\right) d y, \quad z \in \mathbb{C} \backslash(-\infty, q], \\
& \phi_{3}(z)=\frac{1}{2} \int_{-r_{3}}^{z}\left(\xi_{3}(y)-\xi_{4}(y)\right) d y, \quad z \in \mathbb{C} \backslash\left(\left(-\infty,-r_{3}\right] \cup[p, \infty)\right) .
\end{aligned}
$$

\section{Steepest descent analysis for $Y(z)$}

In this section, we will perform the steepest descent analysis of the $\mathrm{RH}$ problem for $Y$. It consists of a series of explicit and invertible transformations. In Section 6, these transformations will be used to prove Theorem 2.3.

To this end, we mainly follow the theme laid out in [38], although the argument is somehow more involved since our RH problem is of size $4 \times 4$ whereas the dimension treated in [38] is $3 \times 3$. Furthermore, we have more generic cases to consider here.

\subsection{First transformation $Y \mapsto X$}

It is the aim of this transformation to simplify the block matrix (2.30) appearing in the jump condition (2.29) for $Y$. The idea is to use the special properties of modified Bessel functions, in a very similar way as in [38]. To start with, we set four functions:

$$
\begin{array}{ll}
y_{1}(z)=z^{(\alpha+1) / 2} I_{\alpha+1}(2 \sqrt{z}), & y_{2}(z)=z^{(\alpha+1) / 2} K_{\alpha+1}(2 \sqrt{z}), \\
y_{3}(z)=z^{-(\alpha-1) / 2} I_{\alpha-1}(2 \sqrt{z}), & y_{4}(z)=z^{-(\alpha-1) / 2} K_{\alpha-1}(2 \sqrt{z}),
\end{array}
$$

where $K_{\nu}$ is the modified Bessel function of the second kind; see [1, Section 9.6] for its main properties. It is readily seen that $y_{3}(z)$ is well defined in the whole complex plane, while the function $y_{j}(z), j=1,2,4$ is analytic in the complex plane with a branch cut along the negative real axis. Indeed, it follows from formulas 9.6.30-9.6.31 in [1] that their jumps on $\mathbb{R}^{-}$are given in the following ways

$$
\begin{aligned}
& y_{1,+}(x)=e^{2 i \alpha \pi} y_{1,-}(x), \\
& y_{2,+}(x)=y_{2,-}(x)+i \pi e^{i \alpha \pi} y_{1,-}(x), \\
& y_{4,+}(x)=e^{-2 i \alpha \pi} y_{4,-}(x)+i \pi e^{-i \alpha \pi} y_{3}(x),
\end{aligned}
$$

for $x<0$. In view of the derivative properties of the modified Bessel functions [1, 9.6.26], we also have

$$
\begin{array}{ll}
y_{1}^{\prime}(z)=z^{\alpha / 2} I_{\alpha}(2 \sqrt{z}), & y_{2}^{\prime}(z)=-z^{\alpha / 2} K_{\alpha}(2 \sqrt{z}), \\
y_{3}^{\prime}(z)=z^{-\alpha / 2} I_{\alpha}(2 \sqrt{z}), & y_{4}^{\prime}(z)=-z^{-\alpha / 2} K_{\alpha}(2 \sqrt{z}) .
\end{array}
$$


A combination of (5.1), (5.3) and (2.30) implies that we can represent the weight matrix $W(x)$ in terms of the functions $y_{1}, y_{3}$ and their derivatives as

$$
\begin{aligned}
& W(x)=e^{-\frac{n x}{t(1-t)}}\left(\begin{array}{c}
x^{\alpha / 2} I_{\alpha}\left(\frac{2 n \sqrt{a x}}{t}\right) \\
x^{(\alpha+1) / 2} I_{\alpha+1}\left(\frac{2 n \sqrt{a x}}{t}\right)
\end{array}\right)\left(x^{-\alpha / 2} I_{\alpha}\left(\frac{2 n \sqrt{b x}}{1-t}\right) \quad x^{-(\alpha-1) / 2} I_{\alpha-1}\left(\frac{2 n \sqrt{b x}}{1-t}\right)\right)
\end{aligned}
$$

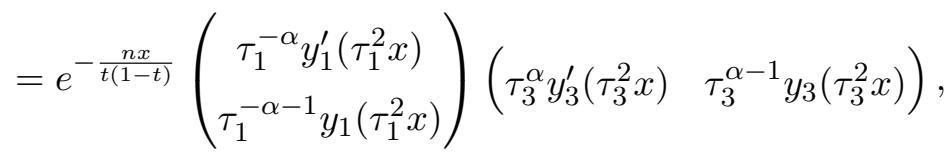

where

$$
\tau_{1}=\tau_{1, n}=\frac{n \sqrt{a}}{t}, \quad \tau_{3}=\tau_{3, n}=\frac{n \sqrt{b}}{1-t} .
$$

For later use, we also need the following Wronskian relations:

$$
y_{1}^{\prime}(z) y_{2}(z)-y_{1}(z) y_{2}^{\prime}(z)=z^{\alpha} / 2, \quad y_{3}^{\prime}(z) y_{4}(z)-y_{3}(z) y_{4}^{\prime}(z)=z^{-\alpha} / 2,
$$

for $z \in \mathbb{C} \backslash \mathbb{R}^{-}$; see formula 9.6 .15 of $[1]$.

The first transformation $Y \mapsto X$ is then defined by (compare with [38])

$$
X(z)=C_{1} Y(z) \operatorname{diag}\left(A_{1}(z), A_{2}(z)\right),
$$

where

$$
\begin{aligned}
& A_{1}(z)=\tau_{1}^{-\alpha} z^{-\alpha / 2}\left(\begin{array}{cc}
-\frac{1}{\pi i} y_{2}^{\prime}\left(\tau_{1}^{2} z\right) & y_{1}^{\prime}\left(\tau_{1}^{2} z\right) \\
-\frac{1}{\pi i} \tau_{1}^{-1} y_{2}\left(\tau_{1}^{2} z\right) & \tau_{1}^{-1} y_{1}\left(\tau_{1}^{2} z\right)
\end{array}\right), \\
& A_{2}(z)=2 \tau_{3}^{\alpha} z^{\alpha / 2}\left(\begin{array}{cc}
y_{4}\left(\tau_{3}^{2} z\right) & -\pi i y_{3}\left(\tau_{3}^{2} z\right) \\
-\tau_{3} y_{4}^{\prime}\left(\tau_{3}^{2} z\right) & \pi i \tau_{3} y_{3}^{\prime}\left(\tau_{3}^{2} z\right)
\end{array}\right),
\end{aligned}
$$

and with $C_{1}$ the constant $4 \times 4$ matrix

$$
C_{1}=\operatorname{diag}\left(\sqrt{2 \pi \tau_{1}}\left(\begin{array}{cc}
i & 0 \\
\frac{4(\alpha+1)^{2}-1}{16 \tau_{1}} & 1
\end{array}\right), \frac{1}{\sqrt{2 \pi \tau_{3}}}\left(\begin{array}{cc}
0 & 1 \\
i & -i \frac{4(\alpha-1)^{2}-1}{16 \tau_{3}}
\end{array}\right)\right) .
$$

Note that, on account of (5.6), we have

$$
\operatorname{det} A_{1}=-\frac{1}{2 \pi \tau_{1}} i, \quad \operatorname{det} A_{2}=2 \pi \tau_{3} i .
$$

Hence, it is easily seen that $\operatorname{det} X=1$. Now, we have

Proposition 5.1. The matrix valued function $X(z)$ defined by (5.7) is the unique solution of the following $R H$ problem. 
(1) $X(z)$ is analytic in $\mathbb{C} \backslash \mathbb{R}$.

(2) For $x \in \mathbb{R}, X(x)$ satisfies the following jump condition

$$
X_{+}(x)=X_{-}(x) \begin{cases}I+e^{-\frac{n x}{t(1-t)}} E_{23}, & \text { if } x>0, \\ \operatorname{diag}\left(e^{-i \pi \alpha}, e^{i \pi \alpha}, e^{-i \pi \alpha}, e^{i \pi \alpha}\right)-E_{21}-E_{43}, & \text { if } x<0,\end{cases}
$$

where we denote by $E_{i j}$ the $4 \times 4$ elementary matrix whose entries are all 0 , except for the $(i, j)$-th entry, which is 1.

(3) As $z \rightarrow \infty, z \in \mathbb{C} \backslash \mathbb{R}$, we have

$$
\begin{aligned}
X(z)= & \left(I+O\left(\frac{1}{z}\right)\right) \operatorname{diag}\left(z^{-1 / 4}, z^{1 / 4}, z^{-1 / 4}, z^{1 / 4}\right) \frac{1}{\sqrt{2}} \operatorname{diag}\left(\left(\begin{array}{cc}
1 & i \\
i & 1
\end{array}\right),\left(\begin{array}{cc}
1 & i \\
i & 1
\end{array}\right)\right) \\
& \times \operatorname{diag}\left(z^{n / 2} e^{-2 n \sqrt{a z} / t}, z^{n / 2} e^{2 n \sqrt{a z} / t}, z^{-n / 2} e^{-2 n \sqrt{b z} /(1-t)}, z^{-n / 2} e^{2 n \sqrt{b z} /(1-t)}\right),
\end{aligned}
$$

uniformly valid for $z$ bounded away from the negative real axis.

(4) $X(z)$ has the following behavior near the origin:

$$
\begin{array}{ll}
\left.X(z) \operatorname{diag}\left(|z|^{\alpha / 2},|z|^{-\alpha / 2},|z|^{\alpha / 2},|z|^{-\alpha / 2}\right)\right)=O(1), & \text { if } \alpha>0, \\
X(z) \operatorname{diag}\left((\log |z|)^{-1}, 1,(\log |z|)^{-1}, 1\right)=O(1), & \text { if } \alpha=0, \\
X(z)=O\left(z^{\alpha / 2}\right), \quad X^{-1}(z)=O\left(z^{\alpha / 2}\right), & \text { if }-1<\alpha<0 .
\end{array}
$$

Proof. The proof follows [38]. It is easily seen from (5.7)-(5.9) and (2.29) that

$$
\begin{gathered}
X_{-}^{-1}(x) X_{+}(x)=\left(\begin{array}{cc}
A_{1,-}^{-1}(x) & 0 \\
0 & A_{2,-}^{-1}(x)
\end{array}\right) Y_{-}^{-1}(x) Y_{+}(x)\left(\begin{array}{cc}
A_{1,+}(x) & 0 \\
0 & A_{2,+}(x)
\end{array}\right) \\
=\left\{\begin{array}{cc}
\left(\begin{array}{cc}
I_{2} & A_{1,-}^{-1}(x) W(x) A_{2,+}(x) \\
0 & I_{2}
\end{array}\right), & \text { if } x>0, \\
\left(\begin{array}{cc}
A_{1,-}^{-1}(x) A_{1,+}(x) & 0 \\
0 & A_{2,-}^{-1}(x) A_{2,+}(x)
\end{array}\right), & \text { if } x<0 .
\end{array}\right.
\end{gathered}
$$

In view of (5.4) and (5.6), we obtain from a direct calculation that

$$
\begin{aligned}
& A_{1,-}^{-1}(x) W(x) A_{2,+}(x) \\
& =e^{-\frac{n x}{(1-t) t}} A_{1,-}^{-1}(x)\left(\begin{array}{c}
\tau_{1}^{-\alpha} y_{1}^{\prime}\left(\tau_{1}^{2} x\right) \\
\tau_{1}^{-\alpha-1} y_{1}\left(\tau_{1}^{2} x\right)
\end{array}\right)\left(\tau_{3}^{\alpha} y_{3}^{\prime}\left(\tau_{3}^{2} x\right)\right. \\
& \left.\tau_{3}^{\alpha-1} y_{3}\left(\tau_{3}^{2} x\right)\right) A_{2,+}(x) \\
& =e^{-\frac{n x}{(1-t) t}}\left(\begin{array}{c}
0 \\
x^{\alpha / 2}
\end{array}\right)\left(\begin{array}{ll}
x^{-\alpha / 2} & 0
\end{array}\right)=e^{-\frac{n x}{(1-t) t}}\left(\begin{array}{ll}
0 & 0 \\
1 & 0
\end{array}\right)
\end{aligned}
$$


for $x>0$. Also, by (5.2) and (5.3), it can be shown that

$$
A_{1,-}^{-1}(x) A_{1,+}(x)=\left(\begin{array}{cc}
e^{-i \pi \alpha} & 0 \\
-1 & e^{i \pi \alpha}
\end{array}\right) \quad \text { and } \quad A_{2,-}^{-1}(x) A_{2,+}(x)=\left(\begin{array}{cc}
e^{-i \pi \alpha} & 0 \\
-1 & e^{i \pi \alpha}
\end{array}\right) \text {, }
$$

for $x<0$. The jump condition (5.11) now follows from a combination of (5.13)-(5.15).

The large $z$ behavior of $X(z)$ shown in item (3), follows very similarly as in [38]. The relevant results are

$$
A_{1}(z)=\frac{1}{\sqrt{2 \pi \tau_{1}}}\left[\left(\begin{array}{cc}
-i & 0 \\
i \frac{4(\alpha+1)^{2}-1}{16 \tau_{1}} & 1
\end{array}\right)+O\left(z^{-1}\right)\right] \frac{z^{-\sigma_{3} / 4}}{\sqrt{2}}\left(\begin{array}{ll}
1 & i \\
i & 1
\end{array}\right) e^{-2 \tau_{1} \sqrt{z} \sigma_{3}}
$$

and

$$
A_{2}(z)=\sqrt{2 \pi \tau_{3}}\left[\left(\begin{array}{cc}
\frac{4(\alpha-1)^{2}-1}{16 \tau_{3}} & -i \\
1 & 0
\end{array}\right)+O\left(z^{-1}\right)\right] \frac{z^{-\sigma_{3} / 4}}{\sqrt{2}}\left(\begin{array}{cc}
1 & i \\
i & 1
\end{array}\right) e^{-2 \tau_{3} \sqrt{z} \sigma_{3}}
$$

as $z \rightarrow \infty$, uniformly for $z$ bounded away from the negative real axis. Substituting the asymptotic formulas (5.16) and (5.17) of $A_{1}(z)$ and $A_{2}(z)$ into (5.7), the asymptotic behavior of $X(z)$ at infinity is immediate.

Finally, the known behavior of the modified Bessel functions near zero given by formulas 9.6.7-9.6.9 in [1] yields

$$
\begin{aligned}
& y_{1}(z) \sim \frac{1}{\Gamma(\alpha+2)} z^{\alpha+1}, \quad y_{1}^{\prime}(z) \sim \frac{1}{\Gamma(\alpha+1)} z^{\alpha}, \\
& y_{2}(z) \sim \frac{1}{2} \Gamma(\alpha+1), \quad y_{2}^{\prime}(z) \sim \begin{cases}-\frac{1}{2} \Gamma(\alpha), & \alpha>0, \\
\frac{1}{2} \log (z), & \alpha=0, \\
-\frac{1}{2} \Gamma(-\alpha) z^{\alpha}, & \alpha<0,\end{cases}
\end{aligned}
$$

and

$$
\begin{aligned}
& y_{3}(z) \sim \frac{1}{\Gamma(\alpha)}, \\
& y_{3}^{\prime}(z) \sim \frac{1}{\Gamma(\alpha+1)}, \\
& y_{4}(z) \sim \begin{cases}\frac{1}{2} \Gamma(\alpha-1) z^{1-\alpha}, & \alpha>1, \\
-\frac{1}{2} \log (z), & \alpha=1, \\
\frac{1}{2} \Gamma(1-\alpha), & \alpha<1,\end{cases} \\
& y_{4}^{\prime}(z) \sim \begin{cases}-\frac{1}{2} \Gamma(\alpha) z^{-\alpha}, & \alpha>0, \\
\frac{1}{2} \log (z), & \alpha=0, \\
-\frac{1}{2} \Gamma(-\alpha), & \alpha<0 .\end{cases}
\end{aligned}
$$

The behavior at zero of $X(z)$ in item (4) then follows from a straightforward calculation.

This completes the proof of Proposition 5.1. 


\subsection{Second transformation $X \mapsto U$}

The second transformation $X \mapsto U$ is defined by

$$
U(z)=C_{2} X(z) \operatorname{diag}\left(e^{n\left(\lambda_{1}(z)-\frac{z}{t(1-t)}\right)}, e^{n\left(\lambda_{2}(z)-\frac{z}{t(1-t)}\right)}, e^{n \lambda_{3}(z)}, e^{n \lambda_{4}(z)}\right),
$$

where $C_{2}$ is the constant matrix

$$
C_{2}=\left(I+2 i n c_{1} E_{21}+2 i n c_{3} E_{34}\right) \operatorname{diag}\left(e^{n l}, e^{n l}, 1,1\right),
$$

with $c_{1}$ and $c_{3}$ as in Lemma 4.2 and with $l$ the variational constant in Proposition 2.2.

In view of the jump relations (5.11) for $X$ and the definition (5.18), we see that the jump relations for $U$ are given by

$$
U_{+}=U_{-} \begin{cases}\Lambda_{-}^{-1}\left(I+E_{23}\right) \Lambda_{+}, & \text {on } \mathbb{R}^{+}, \\ \Lambda_{-}^{-1}\left(\operatorname{diag}\left(e^{-i \pi \alpha}, e^{i \pi \alpha}, e^{-i \pi \alpha}, e^{i \pi \alpha}\right)-E_{21}-E_{43}\right) \Lambda_{+}, & \text {on } \mathbb{R}^{-},\end{cases}
$$

where we define the diagonal matrix

$$
\Lambda(z)=\operatorname{diag}\left(e^{n \lambda_{1}(z)}, e^{n \lambda_{2}(z)}, e^{n \lambda_{3}(z)}, e^{n \lambda_{4}(z)}\right) .
$$

The jump matrix for $U$ can be reformulated with the help of the $\phi$-functions in (4.38)-(4.40) as stated in the following proposition.

Proposition 5.2. The matrix valued function $U(z)$ defined by (5.18) is the unique solution of the following $R H$ problem.

(1) $U(z)$ is analytic in $\mathbb{C} \backslash \mathbb{R}$.

(2) For $x \in \mathbb{R}$, we have that $U_{+}=U_{-} J_{U}$, where

$$
\begin{aligned}
& J_{U}=\operatorname{diag}\left(1,\left(\begin{array}{cc}
e^{2 n \phi_{2,+}} & 1 \\
0 & e^{2 n \phi_{2,-}}
\end{array}\right), 1\right) \quad \text { on } \Delta_{2}, \\
& J_{U}=I+e^{-2 n \phi_{2,+}} E_{23} \quad \text { on } \mathbb{R}^{+} \backslash\left(0 \cup \overline{\Delta_{2}}\right) \text {, } \\
& J_{U}=\operatorname{diag}\left(\left(\begin{array}{cc}
e^{-i \pi \alpha} e^{2 n \phi_{1,+}} & 0 \\
-1 & e^{i \pi \alpha} e^{2 n \phi_{1,-}}
\end{array}\right),\left(\begin{array}{cc}
e^{-i \pi \alpha} e^{2 n \phi_{3,+}} & 0 \\
-1 & e^{i \pi \alpha} e^{2 n \phi_{3,-}}
\end{array}\right)\right) \text { on } \Delta_{3} \text {, } \\
& J_{U}=\left(\left(\begin{array}{cc}
e^{-i \pi \alpha} e^{2 n \phi_{1,+}} & 0 \\
-1 & e^{i \pi \alpha} e^{2 n \phi_{1,-}}
\end{array}\right)\left(\begin{array}{cc}
e^{-i \pi \alpha} & 0 \\
-e^{2 n \phi_{3}} & e^{i \pi \alpha}
\end{array}\right)\right) \text { on } \Delta_{1} \backslash \overline{\Delta_{3}}, \\
& J_{U}=\operatorname{diag}\left(e^{-i \pi \alpha}, e^{i \pi \alpha}, e^{-i \pi \alpha}, e^{i \pi \alpha}\right)-e^{2 n \phi_{1}} E_{21}-e^{2 n \phi_{3}} E_{43} \quad \text { on } \mathbb{R}^{-} \backslash \overline{\Delta_{1}} \text {, }
\end{aligned}
$$


(3) As $z \rightarrow \infty, z \in \mathbb{C} \backslash \mathbb{R}$, we have that

$$
U(z)=\left(I+O\left(\frac{1}{z}\right)\right) \operatorname{diag}\left(z^{-1 / 4}, z^{1 / 4}, z^{-1 / 4}, z^{1 / 4}\right) \frac{1}{\sqrt{2}} \operatorname{diag}\left(\left(\begin{array}{ll}
1 & i \\
i & 1
\end{array}\right),\left(\begin{array}{ll}
1 & i \\
i & 1
\end{array}\right)\right)
$$

uniformly valid for $z$ bounded away from the negative real axis.

(4) $U(z)$ has the same behavior as $X(z)$ near the origin; see (5.12).

Before proceeding to the proof of Proposition 5.2, we remind the reader that, due to our assumption (2.13), the jump condition (5.25) only appears in Cases II and III, while (5.26) only appears in Case III.

Proof. The jump conditions in item (2) are obtained in a straightforward way from Lemma 4.7 and the definitions of $\phi$-functions in (4.38)-(4.40). For convenience, we give a proof of (5.22), the other jumps can be proved in similar ways.

If $x \in \Delta_{2}$, it follows from (5.20) and Lemma 4.7 that

$$
J_{U}(x)=\operatorname{diag}\left(1,\left(\begin{array}{cc}
e^{n\left(\lambda_{2,+}-\lambda_{2,-}\right)(x)} & 1 \\
0 & e^{n\left(\lambda_{3,+}-\lambda_{3,-}\right)(x)}
\end{array}\right), 1\right) .
$$

On account of our definition of $\lambda_{2,3}$ in (4.35) and (4.36), it suffices to show that

$$
g_{2,+}(x)-g_{2,-}(x)=-2 \phi_{2,+}(x)=2 \phi_{2,-}(x),
$$

for $x \in \Delta_{2}$. To see this, we obtain from (4.28) and (4.15) that

$$
g_{2,+}(x)-g_{2,-}(x)=2 \pi i \int_{x}^{q} d \mu_{2}(s)=\int_{q}^{x}\left(\xi_{2,-}(s)-\xi_{2,+}(s)\right) d s .
$$

The relation (5.29) now is immediate, with the aid of (5.30) and the fact that $\xi_{2,-}(s)=$ $\xi_{3,+}(s)$ provided $s \in \Delta_{2}$.

To prove the asymptotic behavior of $U$ near infinity, we note that the asymptotics of $X$ and the large $z$ behavior of the $\lambda$-functions given in Lemma 4.8 yield

$$
\begin{aligned}
& X(z) \operatorname{diag}\left(e^{n\left(\lambda_{1}(z)-\frac{z}{t(1-t)}\right)}, e^{n\left(\lambda_{2}(z)-\frac{z}{t(1-t)}\right)}, e^{n \lambda_{3}(z)}, e^{n \lambda_{4}(z)}\right) \\
& =\left(I+O\left(\frac{1}{z}\right)\right) \operatorname{diag}\left(z^{-1 / 4}, z^{1 / 4}, z^{-1 / 4}, z^{1 / 4}\right) \frac{1}{\sqrt{2}} \operatorname{diag}\left(\left(\begin{array}{ll}
1 & i \\
i & 1
\end{array}\right),\left(\begin{array}{ll}
1 & i \\
i & 1
\end{array}\right)\right) C(z),
\end{aligned}
$$

as $z \rightarrow \infty$, where

$$
C(z)=\operatorname{diag}\left(e^{-n l}\left(1-\frac{2 n c_{1}}{z^{1 / 2}}\right), e^{-n l}\left(1+\frac{2 n c_{1}}{z^{1 / 2}}\right), 1-\frac{2 n c_{3}}{z^{1 / 2}}, 1+\frac{2 n c_{3}}{z^{1 / 2}}\right)+O\left(\frac{1}{z}\right),
$$


where the matrix denoted with $O\left(\frac{1}{z}\right)$ is diagonal as well.

Now we can move $C(z)$ in (5.31) to the left as in the proof of Proposition 5.1. The result is that $(5.31)$ is equal to

$$
C_{2}^{-1}\left(I+O\left(\frac{1}{z}\right)\right) \operatorname{diag}\left(z^{-1 / 4}, z^{1 / 4}, z^{-1 / 4}, z^{1 / 4}\right) \frac{1}{\sqrt{2}} \operatorname{diag}\left(\left(\begin{array}{ll}
1 & i \\
i & 1
\end{array}\right),\left(\begin{array}{ll}
1 & i \\
i & 1
\end{array}\right)\right) .
$$

Then (5.27) is immediate by the definition of $U$ in (5.18).

The behavior of $U$ near the origin follows from the corresponding behavior of $X$, and the fact that the $\lambda$-functions are bounded at the origin.

\subsection{Third transformation $U \mapsto T$}

Our next transformation involves factorizations of jump matrices and the so-called lens opening, which is a key ingredient of the steepest descent analysis. The main goal of this step is to convert the highly oscillatory jump matrices into a more convenient form (e.g., constant matrices) on the original contour, thereby introducing extra jump matrices with exponentially small off-diagonal entries on the new contours.

The existence of lenses is guaranteed by the next lemma.

Lemma 5.3. (Lenses around $\Delta_{j}$.) There exist Jordan curves $\Delta_{2}^{+}, \Delta_{2}^{-}$connecting $p$ to $q$ in the upper and lower half plane, respectively, such that $\operatorname{Re} \phi_{2}(z)<0$ for every non-real $z$ in the region enclosed by $\Delta_{2}^{+}, \Delta_{2}^{-}$. This region is called the lens around $\Delta_{2}$.

Similarly, for $j=1,3$ there exist Jordan curves $\Delta_{j}^{+}, \Delta_{j}^{-}$connecting $-\infty$ to $-r_{j}$ in the upper and lower half plane, respectively, such that $\operatorname{Re} \phi_{j}(z)>0$ for every non-real $z$ in the region enclosed by $\Delta_{j}^{+}, \Delta_{j}^{-}, j=1,3$. This region is called the lens around $\Delta_{j}$. If we are in Case I or $r_{3}=r_{1}$ in Case III, then we may (and do) assume that $\Delta_{3}^{ \pm}=\Delta_{1}^{ \pm}$.

Proof. We first prove the claim for $\Delta_{2}$. Recalling that $\xi_{2,+}(x)=\xi_{3,-}(x)$ for $x \in \Delta_{2}$, it follows from (4.15) and the definition (4.39) of $\phi_{2}(x)$ that

$$
\phi_{2,+}(x)=-\phi_{2,-}(x)=\pi i \int_{q}^{x} d \mu_{2}, \quad x \in \Delta_{2} .
$$

Hence, $\phi_{2, \pm}$ is purely imaginary on $\Delta_{2}$. Furthermore, by differentiating the above formula with respect to $x$ on both sides, we obtain

$$
\frac{d}{d x} \operatorname{Im} \phi_{2,+}(x)>0 \text { and } \frac{d}{d x} \operatorname{Im} \phi_{2,-}(x)<0 .
$$

Therefore, an appeal to the Cauchy-Riemann equations yields the existence of a region around $\Delta_{2}$, such that $\operatorname{Re} \phi_{2}(z)<0$ for every $z \notin\{p, q\}$ in that region.

To prove the claim for $\Delta_{1}$, note that $\xi_{1,+}(x)=\xi_{2,-}(x)$ for $x \in \Delta_{1}$. Then we obtain from (4.38) and (4.14) that

$$
\phi_{1,+}(x)=-\phi_{1,-}(x)=\pi i \int_{-r_{1}}^{x} d\left(\mu_{1}-\rho_{1}\right), \quad x \in \Delta_{1} .
$$




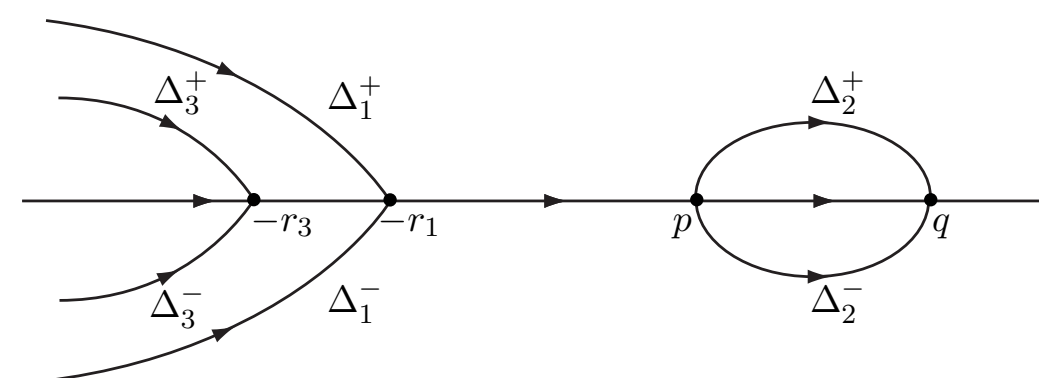

Figure 5: Lenses around $\Delta_{j}, j=1,2,3$. In Case I or $r_{3}=r_{1}$ in Case III, we have $\Delta_{1}^{ \pm}=\Delta_{3}^{ \pm}$.

In view of the constraint condition $\mu_{1} \leq \rho_{1}$, it is immediate that

$$
\frac{d}{d x} \operatorname{Im} \phi_{1,+}(x)<0 \quad \text { and } \quad \frac{d}{d x} \operatorname{Im} \phi_{1,-}(x)>0 .
$$

Again, by the Cauchy-Riemann equations, our claim holds. The claim for $\Delta_{3}$ can be proved similarly.

An illustration of Lemma 5.3 is shown in Figure 5.

The $2 \times 2$ middle block of the jump matrix (5.22) on $\Delta_{2}$ admits the factorization

$$
\left(\begin{array}{cc}
e^{2 n \phi_{2,+}} & 1 \\
0 & e^{2 n \phi_{2,-}}
\end{array}\right)=\left(\begin{array}{cc}
1 & 0 \\
e^{2 n \phi_{2,-}} & 1
\end{array}\right)\left(\begin{array}{cc}
0 & 1 \\
-1 & 0
\end{array}\right)\left(\begin{array}{cc}
1 & 0 \\
e^{2 n \phi_{2,+}} & 1
\end{array}\right) .
$$

Similarly, for the $2 \times 2$ upper left and lower right blocks of the jump matrix (5.24) on $\Delta_{1}$ and $\Delta_{3}$ we have the factorizations

$$
\begin{aligned}
\left(\begin{array}{cc}
e^{-i \pi \alpha} e^{2 n \phi_{j,+}} & 0 \\
-1 & e^{i \pi \alpha} e^{2 n \phi_{j,-}}
\end{array}\right)= & \left(\begin{array}{cc}
1 & -e^{-i \pi \alpha} e^{-2 n \phi_{j,-}} \\
0 & 1
\end{array}\right)\left(\begin{array}{cc}
0 & 1 \\
-1 & 0
\end{array}\right) \\
& \times\left(\begin{array}{cc}
1 & -e^{i \pi \alpha} e^{-2 n \phi_{j,+}} \\
0 & 1
\end{array}\right), \quad j=1,3 .
\end{aligned}
$$

Based on the factorizations (5.36)-(5.37), we define

$$
T(z)=U(z)\left(I \mp e^{2 n \phi_{2}(z)} E_{32}\right),
$$

for $z$ in the domain bounded by $\Delta_{2}^{ \pm}$and $\Delta_{2}$;

$$
T(z)=U(z)\left(I \pm e^{ \pm i \pi \alpha} e^{-2 n \phi_{1}(z)} E_{12}\right),
$$


for $z$ in the domain bounded by $\Delta_{1}^{ \pm}, \Delta_{3}^{ \pm}$and $\Delta_{1}$;

$$
T(z)=U(z)\left(I \pm e^{ \pm i \pi \alpha} e^{-2 n \phi_{1}(z)} E_{12} \pm e^{ \pm i \pi \alpha} e^{-2 n \phi_{3}(z)} E_{34}\right)
$$

for $z$ in the domain bounded by $\Delta_{3}^{ \pm}$and $\Delta_{3}$, and we let

$$
T(z)=U(z),
$$

for $z$ outside of the lenses.

It then follows from straightforward calculations that $T(z)$ is characterized by the following $\mathrm{RH}$ problem:

Proposition 5.4. The matrix valued function $T(z)$ is the unique solution of the following RH problem.

(1) $T$ is analytic in $\mathbb{C} \backslash\left(\mathbb{R} \cup \Delta_{1}^{ \pm} \cup \Delta_{2}^{ \pm} \cup \Delta_{3}^{ \pm}\right)$.

(2) For each of the oriented contours shown in Figure 5, $T$ has a jump $T_{+}=T_{-} J_{T}$, where

$$
J_{T}=\operatorname{diag}\left(1,\left(\begin{array}{cc}
0 & 1 \\
-1 & 0
\end{array}\right), 1\right) \text {, on } \Delta_{2},
$$

$J_{T}=I+e^{-2 n \phi_{2,+}} E_{23}$, on $\mathbb{R}^{+} \backslash\left(0 \cup \overline{\Delta_{2}}\right)$,

$J_{T}=\operatorname{diag}\left(e^{-i \pi \alpha}, e^{i \pi \alpha}, e^{-i \pi \alpha}, e^{i \pi \alpha}\right)-e^{2 n \phi_{1}} E_{21}-e^{2 n \phi_{3}} E_{43}$, on $\left(-r_{1}, 0\right)$ (Case III),

$J_{T}=\operatorname{diag}\left(\left(\begin{array}{cc}0 & 1 \\ -1 & 0\end{array}\right),\left(\begin{array}{cc}e^{-i \pi \alpha} & 0 \\ -e^{2 n \phi_{3}} & e^{i \pi \alpha}\end{array}\right)\right)$, on $\Delta_{1} \backslash \overline{\Delta_{3}}$ (Cases II and III),

$J_{T}=\operatorname{diag}\left(\left(\begin{array}{cc}0 & 1 \\ -1 & 0\end{array}\right),\left(\begin{array}{cc}0 & 1 \\ -1 & 0\end{array}\right)\right)$, on $\Delta_{3}$

$J_{T}=I+e^{2 n \phi_{2}} E_{32}$, on $\Delta_{2}^{ \pm}$,

$J_{T}=I-e^{ \pm i \alpha \pi} e^{-2 n \phi_{1}} E_{12}$, on $\Delta_{1}^{ \pm}$,

$J_{T}=I-e^{ \pm i \alpha \pi} e^{-2 n \phi_{3}} E_{34}$, on $\Delta_{3}^{ \pm}$,

$J_{T}=I-e^{ \pm i \alpha \pi} e^{-2 n \phi_{1}} E_{12}-e^{ \pm i \alpha \pi} e^{-2 n \phi_{3}} E_{34}$, on $\Delta_{1}^{ \pm}=\Delta_{3}^{ \pm}$(Case I or $r_{3}=r_{1}$ in Case III).

(3) As $z \rightarrow \infty$, we have

$T(z)=\left(I+O\left(\frac{1}{z}\right)\right) \operatorname{diag}\left(z^{-1 / 4}, z^{1 / 4}, z^{-1 / 4}, z^{1 / 4}\right) \frac{1}{\sqrt{2}} \operatorname{diag}\left(\left(\begin{array}{ll}1 & i \\ i & 1\end{array}\right),\left(\begin{array}{ll}1 & i \\ i & 1\end{array}\right)\right)$,

uniformly for $z \in \mathbb{C} \backslash \mathbb{R}$. 
(4) $T(z)$ has the same behavior near the origin as $U(z)$ (and $X(z)$ ), see (5.12), provided that $z \rightarrow 0$ outside the lenses that end in 0. [The behavior inside the lenses is different but will not be needed for us.]

(5) $T$ is bounded at the finite endpoints of $\Delta_{j}, j=1,2,3$ other than the origin.

Proof. All the properties follow from straightforward calculations.

To show that the asymptotic behavior of $T$ given in item (3) holds uniformly up to the negative real axis, one needs to trace back the transformations $Y \mapsto X \mapsto U \mapsto T$. It turns out the entries of $T$ are actually linear combinations of the modified Bessel functions, whose asymptotic expansions are uniformly valid up to the negative real axis. We omit the details here.

\subsection{Global parametrix}

By the above constructions, it is easily seen from Lemmas 4.8 and 5.3 that the jump matrices $J_{T}$ in the $\mathrm{RH}$ problem for $T(z)$ all tend to the identity matrix exponentially fast as $n \rightarrow \infty$, except for the jump matrices on $\Delta_{j}, j=1,2,3$. Hence, we may approximate $T$ by a solution $N_{\alpha}$ of the following $4 \times 4$ model $\mathrm{RH}$ problem:

(1) $N_{\alpha}$ is analytic in $\mathbb{C} \backslash\left(\overline{\Delta_{1}} \cup \overline{\Delta_{2}} \cup \overline{\Delta_{3}}\right)$.

(2) $N_{\alpha}$ has continuous boundary values on $\Delta_{j}, j=1,2,3$ and satisfies $N_{\alpha,+}=N_{\alpha,-} J_{N_{\alpha}}$, where

$$
J_{N_{\alpha}}=\left\{\begin{aligned}
\operatorname{diag}\left(\left(\begin{array}{cc}
0 & 1 \\
-1 & 0
\end{array}\right),\left(\begin{array}{cc}
0 & 1 \\
-1 & 0
\end{array}\right)\right), & \text { on } \Delta_{3}, \\
\operatorname{diag}\left(\left(\begin{array}{cc}
0 & 1 \\
-1 & 0
\end{array}\right), e^{-i \pi \alpha}, e^{i \pi \alpha}\right), & \text { on } \Delta_{1} \backslash \overline{\Delta_{3}} \text { (Cases II and III), } \\
\operatorname{diag}\left(e^{-i \pi \alpha}, e^{i \pi \alpha}, e^{-i \pi \alpha}, e^{i \pi \alpha}\right), & \text { on }\left(-r_{1}, 0\right) \text { (Case III), } \\
\operatorname{diag}\left(1,\left(\begin{array}{cc}
0 & 1 \\
-1 & 0
\end{array}\right), 1\right), & \text { on } \Delta_{2} .
\end{aligned}\right.
$$

(3) As $z \rightarrow \infty, z \in \mathbb{C} \backslash \mathbb{R}^{-}$,

$$
N_{\alpha}(z)=\left(I+O\left(\frac{1}{z}\right)\right) \operatorname{diag}\left(z^{-1 / 4}, z^{1 / 4}, z^{-1 / 4}, z^{1 / 4}\right) \frac{1}{\sqrt{2}} \operatorname{diag}\left(\left(\begin{array}{ll}
1 & i \\
i & 1
\end{array}\right),\left(\begin{array}{ll}
1 & i \\
i & 1
\end{array}\right)\right) .
$$

We construct the solution to this $\mathrm{RH}$ problem with the help of meromorphic differentials, following the idea in [41]. We will give the construction for Case III; the modifications for Cases I and II will be briefly commented at the end of this section. 
Consider the Riemann surface in Figure 3. Denote with $p, q$ (sheets 2 and 3), $-r_{1}$ (sheets 1 and 2), $-r_{3}$ (sheets 3 and 4 ) the finite branch points. Also denote with $\infty_{1,2}$ the point at infinity that is common to the first and second sheet, with $\infty_{3,4}$ the point at infinity that is common to the third and fourth sheet, and with $0_{1}$ and $0_{4}$ the origin on the first and fourth sheet respectively. Recall that we are working in Case III, so $p=0$ and $\min \left(r_{1}, r_{3}\right)>0$.

Let $\omega_{i}, i=1,2,3,4$ be four meromorphic differentials (of the third kind) on the Riemann surface with simple poles at the above listed points (and nowhere else), and with residues at these points given by the following table:

\begin{tabular}{c|cccccccc}
$x$ & $p$ & $q$ & $-r_{1}$ & $-r_{3}$ & $\infty_{1,2}$ & $\infty_{3,4}$ & $0_{1}$ & $0_{4}$ \\
\hline $\operatorname{Res}\left(\omega_{1}, x\right)$ & $-1 / 2$ & $-1 / 2$ & $-1 / 2$ & $-1 / 2$ & $1 / 2$ & $3 / 2$ & $-\alpha / 2$ & $\alpha / 2$ \\
\hline $\operatorname{Res}\left(\omega_{2}, x\right)$ & $-1 / 2$ & $-1 / 2$ & $-1 / 2$ & $-1 / 2$ & $-1 / 2$ & $5 / 2$ & $-\alpha / 2$ & $\alpha / 2$ \\
\hline $\operatorname{Res}\left(\omega_{3}, x\right)$ & $-1 / 2$ & $-1 / 2$ & $-1 / 2$ & $-1 / 2$ & $3 / 2$ & $1 / 2$ & $-\alpha / 2$ & $\alpha / 2$ \\
\hline $\operatorname{Res}\left(\omega_{4}, x\right)$ & $-1 / 2$ & $-1 / 2$ & $-1 / 2$ & $-1 / 2$ & $5 / 2$ & $-1 / 2$ & $-\alpha / 2$ & $\alpha / 2$
\end{tabular}

The differences among $\omega_{i}$ are that their residues at $\infty_{1,2}$ and $\infty_{3,4}$ are all different. Such meromorphic differentials exist because the sum of the residues is zero. The fact that our Riemann surface has genus 0 implies that each meromorphic differential is unique; cf. $[25,45]$.

We then build the first row of $N_{\alpha}$ with the aid of meromorphic differential $\omega_{1}$ introduced in (5.42). Let $p_{1}$ be an arbitrary reference point on the first sheet of the Riemann surface, chosen on the positive real line such that $p_{1}>q$. Define four functions

$$
u_{j}(z)=\int_{p_{1}}^{z_{j}} \omega_{1}, \quad j=1, \ldots, 4,
$$

where we denote with $z_{j}$ the point $z$ lying on the $j$ th sheet. The integration path from $p_{1}$ to $z_{j}$ obeys the following rules: It can only move from one sheet to another by going from the lower side of $\Delta_{j}$ on sheet $j$ to the upper side of $\Delta_{j}$ on sheet $j+1$. Moreover, the path is not allowed to go through a pole of $\omega_{1}$, and it must not intersect the intervals $\left[-r_{1}, 0\right]$ on the first and second sheets, nor the intervals $\left[-r_{3}, 0\right]$ on the third and fourth sheets, except possibly at the endpoint of the path.

An illustration of the integration path for $u_{3}(z)$ is shown in Figure 6.

With the above conventions, the functions $u_{j}(z)$ satisfy the following jump properties

$$
\begin{aligned}
u_{1,+} & =u_{1,-}+2 \pi i \operatorname{Res}\left(\omega_{1}, 0_{1}\right)=u_{1,-}-\alpha \pi i, & & \text { on }\left(-r_{1}, 0\right), \\
u_{2,+} & =u_{2,-}-2 \pi i\left(\operatorname{Res}\left(\omega_{1}, 0_{1}\right)+\operatorname{Res}\left(\omega_{1}, \infty_{1,2}\right)+\operatorname{Res}\left(\omega_{1},-r_{1}\right)\right) & & \\
& =u_{2,-}+\alpha \pi i, & & \text { on }\left(-r_{1}, 0\right), \\
u_{3,+} & =u_{3,-}-2 \pi i\left(\operatorname{Res}\left(\omega_{1}, 0_{4}\right)+\operatorname{Res}\left(\omega_{1}, \infty_{3,4}\right)+\operatorname{Res}\left(\omega_{1},-r_{3}\right)\right) & & \\
& =u_{3,-}-2 \pi i(1+\alpha / 2), & & \text { on }\left(-r_{3}, 0\right), \\
u_{4,+} & =u_{4,-}+2 \pi i \operatorname{Res}\left(\omega_{1}, 0_{4}\right)=u_{4,-}+\alpha \pi i, & & \text { on }\left(-r_{3}, 0\right) .
\end{aligned}
$$



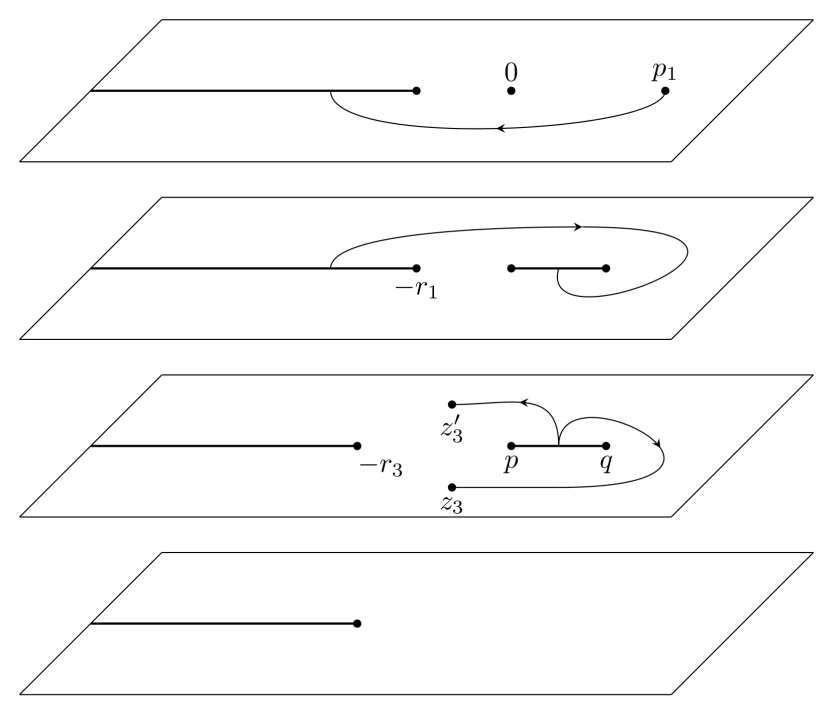

Figure 6: Integration path for $u_{3}(z)$.

Each of these jumps can be easily verified by deforming the integration path of $u_{j,+}-u_{j,-}$ to a closed loop and checking which poles are inside (or outside) the loop. Similarly, we have

$$
\begin{array}{lll}
u_{2,+}=u_{1,-}, & u_{2,-}=u_{1,+}+2 \pi i \operatorname{Res}\left(\omega_{1}, \infty_{1,2}\right)=u_{1,+}+\pi i, & \text { on } \Delta_{1}, \\
u_{3,+}=u_{2,-}, & u_{3,-}=u_{2,+}-2 \pi i \operatorname{Res}\left(\omega_{1}, q\right)=u_{2,+}+\pi i, & \text { on } \Delta_{2}, \\
u_{4,+}=u_{3,-}, & u_{4,-}=u_{3,+}+2 \pi i \operatorname{Res}\left(\omega_{1}, \infty_{3,4}\right)=u_{3,+}+3 \pi i, & \text { on } \Delta_{3} .
\end{array}
$$

Now set

$$
v_{j}(z)=\exp \left(u_{j}(z)\right), \quad j=1, \ldots, 4,
$$

and consider the row vector $\mathbf{v}(z):=\left(v_{1}(z), v_{2}(z), v_{3}(z), v_{4}(z)\right)$. By (5.42)-(5.44) we find that

$$
\mathbf{v}_{+}=\mathbf{v}_{-} J_{N_{\alpha}}, \quad \text { on } \Delta_{1} \cup \Delta_{2} \cup \Delta_{3},
$$

in Case III. Thus $\mathbf{v}(z)$ satisfies the required jumps for the row vectors of $N_{\alpha}$.

Next we discuss the asymptotics of $\mathbf{v}(z)$ for $z \rightarrow \infty$. Taking into account that $\infty_{1,2}$ is a branch point on the Riemann surface, its local coordinate on the Riemann surface can be chosen as $w(z)=1 / \sqrt{z}$. Therefore

$$
\begin{aligned}
u_{j}(z) & =\operatorname{Res}\left(\omega_{1}, \infty_{1,2}\right) \log w(z)+\gamma_{j}+O(w(z)) \\
& =-\frac{1}{4} \log z+\gamma_{j}+O\left(z^{-1 / 2}\right), \quad z \rightarrow \infty,
\end{aligned}
$$


for $j=1,2$, where $\gamma_{1}$ and $\gamma_{2}$ are certain constants. From the relation $u_{2,+}=u_{1,-}$ on $\left(-\infty,-r_{1}\right)$ we find that $\gamma_{2}=\gamma_{1}+\operatorname{Res}\left(\omega_{1}, \infty_{1,2}\right) \pi i=\gamma_{1}+\pi i / 2$. Similarly we also have

$$
u_{j}(z)=-\frac{3}{4} \log z+\gamma_{j}+O\left(z^{-1 / 2}\right), \quad z \rightarrow \infty,
$$

for $j=3,4$, where $\gamma_{3}$ and $\gamma_{4}$ are certain constants. Combining all of this and using (5.42) and (5.45), we find that

$$
\mathbf{v}(z)=\left(e^{\gamma_{1}} z^{-1 / 4}, i e^{\gamma_{1}} z^{-1 / 4}, 0,0\right)+O\left(z^{-3 / 4}\right), \quad z \rightarrow \infty .
$$

The behavior of $\mathbf{v}(z)$ at the origin can be found from similar arguments as above and is given by

$$
\mathbf{v}(z)=O\left(z^{-\alpha / 2}, z^{-1 / 4}, z^{-1 / 4}, z^{\alpha / 2}\right), \quad z \rightarrow 0 .
$$

Finally, we also find that

$$
\mathbf{v}(z)=O\left((|z-\kappa|)^{-1 / 4}\right), \quad \text { as } z \rightarrow \kappa,
$$

if $\kappa$ is a branch point other than the origin.

Summarizing, we see that $\frac{e^{-\gamma_{1}}}{\sqrt{2}} \mathbf{v}(z)$ satisfies all the constraints for the first row of $N_{\alpha}$. The other meromorphic differentials $\omega_{i}, i=2,3,4$ in Table 5.42 can be used to build the $i$-th row of $N_{\alpha}$ in a similar way, we omit the details here.

Our construction of $N_{\alpha}$ leads to

$$
N_{\alpha}(z)=O\left((|z-\kappa|)^{-1 / 4}\right), \quad \text { as } z \rightarrow \kappa,
$$

if $\kappa$ is a branch point other than the origin, and

$$
N_{\alpha}(z) \operatorname{diag}\left(z^{\alpha / 2}, z^{1 / 4}, z^{1 / 4}, z^{-\alpha / 2}\right)=O(1), \quad \text { as } z \rightarrow 0 \quad \text { (Case III). }
$$

Similar constructions can be given in Cases I and II. For instance, we may build the first row of $N_{\alpha}$ in terms of a meromorphic differential $\omega$ with prescribed simple poles and residues as shown in the following tables:

$$
\begin{array}{c|cccccc}
x & p & q & 0_{1,2} & 0_{3,4} & \infty_{1,2} & \infty_{3,4} \\
\hline \operatorname{Res}(\omega, x) & -1 / 2 & -1 / 2 & -1 / 2 & -1 / 2 & 1 / 2 & 3 / 2
\end{array}
$$

for Case I, and

$$
\begin{array}{c|cccccccc}
x & p & q & 0_{1,2} & -r_{3} & \infty_{1,2} & \infty_{3,4} & 0_{3} & 0_{4} \\
\hline \operatorname{Res}(\omega, x) & -1 / 2 & -1 / 2 & -1 / 2 & -1 / 2 & 1 / 2 & 3 / 2 & -\alpha / 2 & \alpha / 2
\end{array}
$$

for Case II. Such constructions will again give us (5.48), but the behavior near the origin is different:

$$
\begin{array}{r}
N_{\alpha}(z)=O\left(z^{-1 / 4}\right), \quad \text { as } z \rightarrow 0 \quad \text { (Case I), } \\
N_{\alpha}(z) \operatorname{diag}\left(z^{1 / 4}, z^{1 / 4}, z^{\alpha / 2}, z^{-\alpha / 2}\right)=O(1), \quad \text { as } z \rightarrow 0 \quad \text { (Case II). }
\end{array}
$$

In what follows, we will construct local parametrices near each branch point. 


\subsection{Parametrix near the nonzero branch points}

Near each of the branch points in $\left\{p, q,-r_{1},-r_{3}\right\} \backslash\{0\}$, we need to build a local parametrix $P^{\text {Airy }}$ using the $2 \times 2 \mathrm{RH}$ problem for Airy functions. This construction is very well-known and we omit the details.

\subsection{Parametrix near the branch point 0 (hard edge)}

The local parametrix at the origin is built by means of modified Bessel functions. Such a construction was given before for a $2 \times 2 \mathrm{RH}$ problem in [40] and in a $3 \times 3$ setting in [38, 43]. Our construction will be similar to the one in [38]. For $-1<\alpha<0$ there are some extra complications that were solved in an ad hoc way in [38]. We will be facing similar problems and solve them in a more conceptual way.

\subsubsection{RH problem for modified Bessel functions}

First we recall the construction of [40]. Define the $2 \times 2$ matrix valued function $\Psi^{\operatorname{Bessel}}(\zeta)$ by

$$
\Psi^{\text {Bessel }}(\zeta)=\left(\begin{array}{cc}
I_{\alpha}\left(2 \zeta^{1 / 2}\right) & \frac{i}{\pi} K_{\alpha}\left(2 \zeta^{1 / 2}\right) \\
2 \pi i \zeta^{1 / 2} I_{\alpha}^{\prime}\left(2 \zeta^{1 / 2}\right) & -2 \zeta^{1 / 2} K_{\alpha}^{\prime}\left(2 \zeta^{1 / 2}\right)
\end{array}\right)
$$

for $|\arg \zeta|<2 \pi / 3$,

$$
\Psi^{\text {Bessel }}(\zeta)=\left(\begin{array}{cc}
\frac{1}{2} H_{\alpha}^{(1)}\left(2(-\zeta)^{1 / 2}\right) & \frac{1}{2} H_{\alpha}^{(2)}\left(2(-\zeta)^{1 / 2}\right) \\
\pi \zeta^{1 / 2}\left(H_{\alpha}^{(1)}\right)^{\prime}\left(2(-\zeta)^{1 / 2}\right) & \pi \zeta^{1 / 2}\left(H_{\alpha}^{(2)}\right)^{\prime}\left(2(-\zeta)^{1 / 2}\right)
\end{array}\right) e^{\frac{i \pi \alpha}{2} \sigma_{3}},
$$

for $2 \pi / 3<\arg \zeta<\pi$, and

$$
\Psi^{\text {Bessel }}(\zeta)=\left(\begin{array}{cc}
\frac{1}{2} H_{\alpha}^{(2)}\left(2(-\zeta)^{1 / 2}\right) & -\frac{1}{2} H_{\alpha}^{(1)}\left(2(-\zeta)^{1 / 2}\right) \\
-\pi \zeta^{1 / 2}\left(H_{\alpha}^{(2)}\right)^{\prime}\left(2(-\zeta)^{1 / 2}\right) & \pi \zeta^{1 / 2}\left(H_{\alpha}^{(1)}\right)^{\prime}\left(2(-\zeta)^{1 / 2}\right)
\end{array}\right) e^{-\frac{i \pi \alpha}{2} \sigma_{3}}
$$

for $-\pi<\arg \zeta<-2 \pi / 3$, where $I_{\alpha}$ and $K_{\alpha}$ are modified Bessel functions and $H_{\alpha}^{(i)}$, $i=1,2$ is the Hankel function; cf. [1, Chapter 9].

Denote with $\gamma_{j}, j=1,2,3$ the complex rays $\{\zeta \in \mathbb{C} \mid \arg \zeta=(j+1) \pi / 3\}$. According to [40], $\Psi^{\text {Bessel }}$ satisfies the following $\mathrm{RH}$ problem:

(1) $\Psi^{\text {Bessel }}$ is analytic in $\mathbb{C} \backslash \bigcup_{j=1}^{3} \gamma_{j}$. 
(2) With the rays $\gamma_{j}, j=1,2,3$ all oriented towards the origin, $\Psi^{\text {Bessel }}$ has the jumps

$$
\Psi_{+}^{\text {Bessel }}=\Psi_{-}^{\text {Bessel }} \times \begin{cases}\left(\begin{array}{cc}
1 & 0 \\
e^{i \pi \alpha} & 1
\end{array}\right), & \text { on } \gamma_{1}, \\
\left(\begin{array}{cc}
0 & 1 \\
-1 & 0
\end{array}\right), & \text { on } \gamma_{2}, \\
\left(\begin{array}{cc}
1 & 0 \\
e^{-i \pi \alpha} & 1
\end{array}\right), & \text { on } \gamma_{3} .\end{cases}
$$

(3) Uniformly for $\zeta \rightarrow \infty$ we have

$$
\Psi^{\mathrm{Bessel}}(\zeta)=\left(2 \pi \zeta^{1 / 2}\right)^{-\sigma_{3} / 2}\left(\frac{1}{\sqrt{2}}\left(\begin{array}{ll}
1 & i \\
i & 1
\end{array}\right)+O\left(\zeta^{-1 / 2}\right)\right) e^{2 \zeta^{1 / 2} \sigma_{3}}
$$

(4) As $\zeta \rightarrow 0$ in $|\arg \zeta|<2 \pi / 3$ we have

$$
\Psi^{\text {Bessel }}(\zeta)=\left\{\begin{array}{cc}
O\left(\begin{array}{cc}
\zeta^{\alpha / 2} & \zeta^{-\alpha / 2} \\
\zeta^{\alpha / 2} & \zeta^{-\alpha / 2}
\end{array}\right), & \text { if } \alpha>0, \\
O\left(\begin{array}{cc}
1 & \log |\zeta| \\
1 & \log |\zeta|
\end{array}\right), & \text { if } \alpha=0, \\
O\left(\zeta^{\alpha / 2}\right), & \text { if } \alpha<0 .
\end{array}\right.
$$

\subsubsection{Construction for $\alpha \geq 0$}

In the construction of the local parametrix at the origin we will restrict ourselves to Case II, which is in some sense the most general and difficult of the three cases. The modifications for Cases I and III will be briefly commented at the end of this section.

We denote by $B_{\delta}$ a small fixed disk with radius $\delta>0$ centered at the origin, such that it does not contain any other branch points and consider all the jump matrices $J_{T}$ of $T$ restricted in $B_{\delta}$; cf. item (2) in Proposition 5.4. We note that the $(2,3)$ entry of $J_{T}$ on $(0, \delta)$ is $e^{-2 n \phi_{2,+}}$. Since $\operatorname{Re} \phi_{2,+}=\operatorname{Re}\left(\lambda_{2}-\lambda_{3}\right)>c>0$ on $(0, \delta)$ (cf. Lemma 4.7), this entry is exponentially small as $n \rightarrow \infty$. Similarly the $(4,3)$ entry of $J_{T}$ on $(-\delta, 0)$ is $-e^{2 n \phi_{3}}$ which is also exponentially small.

By neglecting the exponentially small entries in the jump matrices for $T(z)$ in $B_{\delta}$, we are led to the following $\mathrm{RH}$ problem for a $4 \times 4$ matrix valued function $Q(z)$ :

(1) $Q$ is analytic in $B_{\delta} \backslash\left(\Delta_{1} \cup \Delta_{1}^{ \pm}\right)$. 

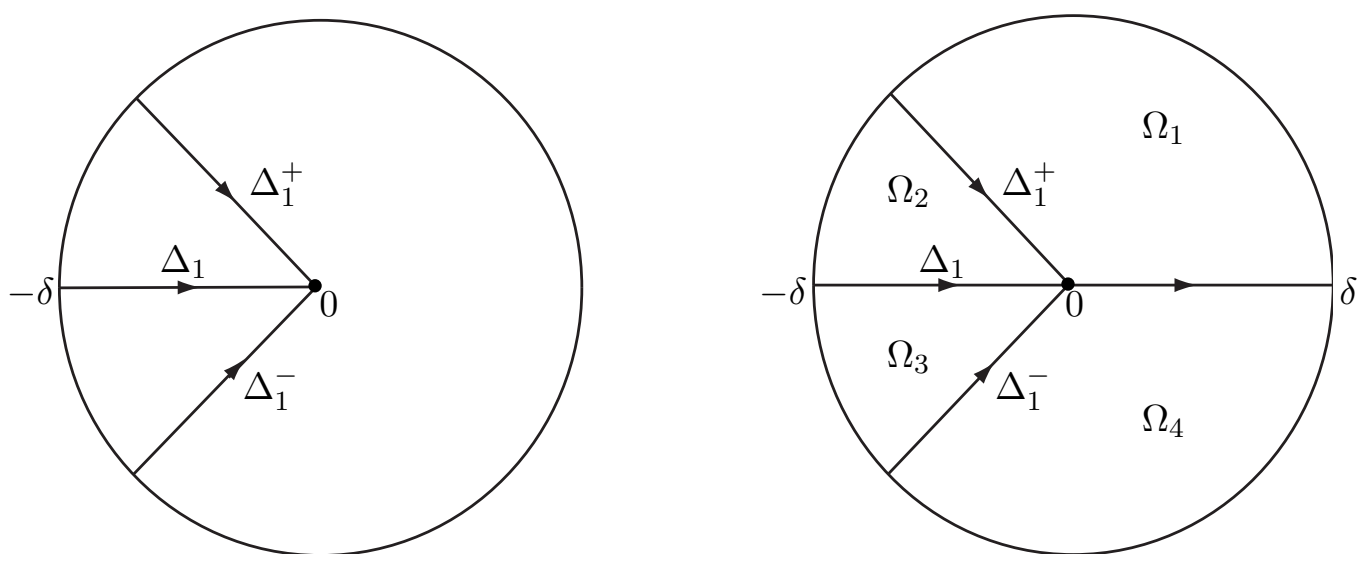

Figure 7: Contours for the local parametrix around 0 in Case II, for $\alpha \geq 0$ (left picture) and for $-1<\alpha<0$ (right picture).

(2) On each of the oriented contours in the left picture of Figure 7, $Q$ has a jump $Q_{+}=Q_{-} J_{Q}$, where

$$
J_{Q}= \begin{cases}\operatorname{diag}\left(\left(\begin{array}{cc}
0 & 1 \\
-1 & 0
\end{array}\right), e^{-i \pi \alpha}, e^{i \pi \alpha}\right), & \text { on } \Delta_{1} \cap B_{\delta}=(-\delta, 0), \\
I-e^{ \pm \alpha \pi i} e^{-2 n \phi_{1}} E_{12}, & \text { on } \Delta_{1}^{ \pm} \cap B_{\delta}\end{cases}
$$

(3) As $z \rightarrow 0$ outside the lens enclosed by $\Delta_{1}^{ \pm}$we have

$$
\begin{array}{ll}
Q(z) \operatorname{diag}\left(|z|^{\alpha / 2},|z|^{-\alpha / 2},|z|^{\alpha / 2},|z|^{-\alpha / 2}\right)=O(1), & \text { if } \alpha>0, \\
Q(z) \operatorname{diag}\left((\log |z|)^{-1}, 1,(\log |z|)^{-1}, 1\right)=O(1), & \text { if } \alpha=0 .
\end{array}
$$

(4) As $n \rightarrow \infty$,

$$
Q(z)=N_{\alpha}(z)\left(I+O\left(\frac{1}{n}\right)\right)
$$

uniformly for $z \in \partial B_{\delta} \backslash\left(\Delta_{1} \cup \Delta_{1}^{ \pm}\right)$, where $N_{\alpha}$ is the global parametrix built in Section 5.4.

Before solving this $\mathrm{RH}$ problem, let us consider the function

$$
f_{1}(z):=\left(\phi_{1}(z)\right)^{2} .
$$

Since the density of $\rho_{1}-\mu_{1}$ blows up like $O\left(|x|^{-1 / 2}\right)$ as $x \rightarrow 0$, we obtain from (4.14) and the definition of $\phi_{1}(z)$ in (4.38) that $f_{1}(z)$ is analytic in $B_{\delta}$ and gives a conformal mapping from a neighborhood of the origin onto itself, such that $f_{1}(x)$ is real and positive 
for $x \in(0, \delta)$. For our purpose, we may deform the contours $\Delta_{1}^{ \pm}$near 0 such that $f_{1}$ maps $\Delta_{1}^{ \pm} \cap B_{\delta}$ to the rays with angles $2 \pi / 3$ and $-2 \pi / 3$, respectively.

We now define

$$
Q(z)=E(z) \operatorname{diag}\left(\sigma_{1} \Psi^{\text {Bessel }}\left(n^{2} f_{1}(z)\right) \sigma_{1}, z^{-\alpha / 2}, z^{\alpha / 2}\right) \operatorname{diag}\left(e^{n \phi_{1}(z)},-e^{-n \phi_{1}(z)}, 1,1\right),
$$

where $\sigma_{1}=\left(\begin{array}{ll}0 & 1 \\ 1 & 0\end{array}\right)$, and where the prefactor $E(z)$ is analytic in $B_{\delta}$ and is chosen to satisfy the matching condition on $\partial B_{\delta}$, see below. With this definition, the items (1), (2) and (3) in the RH problem for $Q(z)$ are satisfied, by virtue of items (1), (2) and (4) in the $\mathrm{RH}$ problem for $\Psi^{\text {Bessel }}(z)$.

To achieve the matching condition in item (4) of the RH problem for $Q(z)$, we take $E(z)$ in (5.59) as

$$
\begin{aligned}
E(z)= & N_{\alpha}(z) \operatorname{diag}(1,-1,1,1) \operatorname{diag}\left(\frac{1}{\sqrt{2}}\left(\begin{array}{cc}
1 & -i \\
-i & 1
\end{array}\right), z^{\alpha / 2}, z^{-\alpha / 2}\right) \\
& \times \operatorname{diag}\left((2 \pi n)^{-1 / 2} f_{1}(z)^{-1 / 4},(2 \pi n)^{1 / 2} f_{1}(z)^{1 / 4}, 1,1\right) .
\end{aligned}
$$

Obviously $E(z)$ is analytic for $z \in B_{\delta} \backslash \mathbb{R}^{-}$. Moreover, one checks that $E(z)$ is also analytic across $(-\delta, 0)$. Finally, since $f_{1}(z)^{1 / 4}=O\left(z^{1 / 4}\right)$ as $z \rightarrow 0$ and using (5.49) we find that

$$
E(z)=O\left(z^{-1 / 2}\right), \quad z \rightarrow 0,
$$

so $E(z)$ cannot have a pole at zero. We conclude that $E(z)$ is analytic in the disk $B_{\delta}$. By virtue of (5.54), the matching condition (5.57) in the RH problem for $Q$ is satisfied.

In summary, we have established the following proposition.

Proposition 5.5. The matrix valued function $Q$ defined by (5.59) and (5.60) satisfies the conditions (1)-(4) of the RH problem for $Q$.

In view of the fact that $\operatorname{det} N_{\alpha}(z)=1$ and $\operatorname{det} \Psi^{\text {Bessel }}=1$ (see [40]), we see that

$$
\operatorname{det} Q(z) \equiv 1, \quad z \in B_{\delta} .
$$

If we take $Q$ as the local parametrix for $T$, the final transformation would be defined as

$$
R(z)=T(z) Q(z)^{-1}, \quad z \in B_{\delta} .
$$

Then $R$ would be analytic in $B_{\delta} \backslash(0, \infty)$ with the following jump on $(0, \delta)$,

$$
\begin{aligned}
R_{-}(x)^{-1} R_{+}(x) & =Q(x) T_{-}(x)^{-1} T_{+}(x) Q(x)^{-1} \\
& =Q(x)\left(I+e^{-2 n \phi_{2,+}(x)} E_{23}\right) Q(x)^{-1} \\
& =I+e^{-2 n \phi_{2,+}(x)} Q(x) E_{23} Q(x)^{-1} .
\end{aligned}
$$

Similarly, the jump on $(-\delta, 0)$ would be

$$
R_{-}(x)^{-1} R_{+}(x)=I-e^{-i \pi \alpha} e^{2 n \phi_{3}(x)} Q_{+}(x) E_{43} Q_{+}(x)^{-1} .
$$


Lemma 5.6. For $\alpha \geq 0$, the matrix $Q(x) E_{23} Q(x)^{-1}$ is bounded as $x \rightarrow 0, x>0$. Similarly, $Q_{+}(x) E_{43} Q_{+}(x)^{-1}$ is bounded as $x \rightarrow 0, x<0$.

Proof. We prove the first statement only; the second follows similarly. Observe that

$$
Q(x) E_{23} Q(x)^{-1}=Q(x)\left(\begin{array}{llll}
0 & 1 & 0 & 0
\end{array}\right)^{T}\left(\begin{array}{llll}
0 & 0 & 1 & 0
\end{array}\right) Q(x)^{-1} .
$$

If $\alpha>0$, it follows from (5.56) and $\operatorname{det} Q(z) \equiv 1$ that both $Q(x)\left(\begin{array}{llll}0 & 1 & 0 & 0\end{array}\right)^{T}$ and $\left(\begin{array}{llll}0 & 0 & 1 & 0\end{array}\right) Q(x)^{-1}$ behave like $O\left(z^{\alpha / 2}\right)$, and the lemma follows.

If $\alpha=0$, we use (5.55) and $\operatorname{det} \Psi^{\text {Bessel }}(\zeta)=1$ to get

$$
\left(\Psi^{\text {Bessel }}\right)^{-1}(\zeta)=\left(\begin{array}{cc}
O(1) & O(1) \\
O(\log |\zeta|) & O(\log |\zeta|)
\end{array}\right) \quad \text { as } \zeta \rightarrow 0 .
$$

Inserting this formula in (5.59), we have

$$
Q^{-1}(x)=\operatorname{diag}\left(\left(\begin{array}{cc}
O(1) & O(1) \\
O(\log x) & O(\log x)
\end{array}\right), O(1), O(1)\right) E^{-1}(x),
$$

as $x \rightarrow 0$ and $x>0$, where $E^{-1}(x)$ is bounded near the origin. Since $Q(x)\left(\begin{array}{llll}0 & 1 & 0 & 0\end{array}\right)^{T}$ is bounded by (5.56) and $\left(\begin{array}{llll}0 & 0 & 1 & 0\end{array}\right) Q(x)^{-1}$ is bounded by $(5.67)$, the lemma follows for $\alpha=0$ as well.

The above lemma, together with (5.64) and the fact that $\operatorname{Re} \phi_{2,+}(x)>c>0$ for some constant $c$ on $(0, \delta)$, implies that, if $\alpha \geq 0$, the jump matrix for $R$ is exponentially close to the identity matrix as $n \rightarrow \infty$, uniformly for $x \in(0, \delta)$. A similar statement holds for the jump on $(-\delta, 0)$. We therefore take the parametrix $P=Q$ in case $\alpha \geq 0$.

\subsubsection{Construction for $-1<\alpha<0$}

If $-1<\alpha<0$, then the $(2,3)$ entry of the jump matrix $J_{T}$ on $(0, \delta)$ and the $(4,3)$ entry of $J_{T}$ on $(-\delta, 0)$ cannot be simplify neglected. Hence, we need to build a $4 \times 4$ matrix valued function $P$ such that

(1) $P$ is analytic in $B_{\delta} \backslash\left(\mathbb{R} \cup \Delta_{1}^{ \pm}\right)$.

(2) For each of the oriented contours shown in the right picture of Figure 7, $P$ has a jump $P_{+}=P_{-} J_{P}$, where

$$
J_{P}= \begin{cases}\operatorname{diag}\left(\left(\begin{array}{cc}
0 & 1 \\
-1 & 0
\end{array}\right),\left(\begin{array}{cc}
e^{-i \pi \alpha} & 0 \\
-e^{2 n \phi_{3}} & e^{i \pi \alpha}
\end{array}\right)\right), & \text { on } \Delta_{1} \cap B_{\delta}=(-\delta, 0), \\
I-e^{ \pm \alpha \pi i} e^{-2 n \phi_{1}} E_{12}, & \text { on } \Delta_{1}^{ \pm} \cap B_{\delta}, \\
I+e^{-2 n \phi_{2,+}} E_{23}, & \text { on }(0, \delta) .\end{cases}
$$


(3) $P(z)$ behaves near the origin like:

$$
P(z)=O\left(z^{\alpha / 2}\right), \quad P^{-1}(z)=O\left(z^{\alpha / 2}\right) .
$$

(4) As $n \rightarrow \infty$,

$$
P(z)=N_{\alpha}(z)\left(I+O\left(\frac{1}{n}\right)\right),
$$

uniformly for $z \in \partial B_{\delta} \backslash\left(\Delta_{1} \cup \Delta_{1}^{ \pm}\right)$, where $N_{\alpha}$ is the global parametrix built in Section 5.4.

We use the matrix valued function $Q$ given by (5.59) and (5.60), which works as the parametrix for the case $\alpha \geq 0$, to construct $P$. More precisely, recall the sectors $\Omega_{j}$, $j=1, \ldots, 4$, in the right picture of Figure 7 . By setting

$$
S(z)=\Lambda^{-1}(z) S_{j}(z) \Lambda(z), \quad z \in \Omega_{j}
$$

where $\Lambda$ is defined in (5.21) and $S_{j}, j=1, \ldots, 4$ is a constant matrix that depends on the sector $\Omega_{j}$, we construct $P$ in the form

$$
P(z)=Q(z) S(z) .
$$

The construction of these four matrices $S_{j}$ is different in the three Cases I-III; we will again explain it for Case II.

Let us give names to the jump matrix $J_{P}$ on the four jump contours around zero as follows. $J_{P, 1}$ is the jump matrix of $P$ on $(0, \delta), J_{P, 2}$ is the jump matrix on $\Delta_{1}^{+}, J_{P, 3}$ on $(-\delta, 0)$ and $J_{P, 4}$ on $\Delta_{1}^{-}$. Similarly we define the jump matrices $J_{Q, k}, k=1, \ldots, 4$. Note that $J_{Q, 2}=J_{P, 2}, J_{Q, 4}=J_{P, 4}, J_{Q, 1}$ differs from $J_{P, 1}$ in the $(2,3)$ entry, and $J_{Q, 3}$ differs from $J_{P, 3}$ in the $(4,3)$ entry,

To obtain the correct jumps for $P$, the matrices $S_{j}$ in (5.70) should be such that

$$
\begin{gathered}
\Lambda J_{P, 1}^{-1} \Lambda^{-1}=S_{1}^{-1}\left(\Lambda J_{Q, 1}^{-1} \Lambda^{-1}\right) S_{4}, \\
\Lambda J_{P, k} \Lambda^{-1}=S_{k}^{-1}\left(\Lambda J_{Q, k} \Lambda^{-1}\right) S_{k-1}, \quad \text { for } k=2,3,4 .
\end{gathered}
$$

Multiplying these equations into a telescoping product form yields the following relation for $S_{1}$ :

$$
\Lambda\left(J_{P, 1}^{-1} J_{P, 4} J_{P, 3} J_{P, 2}\right) \Lambda^{-1}=S_{1}^{-1} \Lambda\left(J_{Q, 1}^{-1} J_{Q, 4} J_{Q, 3} J_{Q, 2}\right) \Lambda^{-1} S_{1} .
$$

Now $\Lambda\left(J_{P, 1}^{-1} J_{P, 4} J_{P, 3} J_{P, 2}\right) \Lambda^{-1}$ and $\Lambda\left(J_{Q, 1}^{-1} J_{Q, 4} J_{Q, 3} J_{Q, 2}\right) \Lambda^{-1}$ are both constant matrices. A calculation shows that they have the spectral decompositions

$$
\begin{gathered}
\Lambda\left(J_{P, 1}^{-1} J_{P, 4} J_{P, 3} J_{P, 2}\right) \Lambda^{-1}=\left(\begin{array}{cccc}
e^{-i \pi \alpha} & 0 & 0 & 0 \\
-1 & e^{i \pi \alpha} & -e^{-i \pi \alpha} & 0 \\
0 & 0 & e^{-i \pi \alpha} & 0 \\
0 & 0 & -1 & e^{i \pi \alpha}
\end{array}\right)=: V_{P} D V_{P}^{-1}, \\
\Lambda\left(J_{Q, 1}^{-1} J_{Q, 4} J_{Q, 3} J_{Q, 2}\right) \Lambda^{-1}=\operatorname{diag}\left(e^{-i \pi \alpha}, e^{i \pi \alpha}, e^{-i \pi \alpha}, e^{i \pi \alpha}\right)-E_{21}=: V_{Q} D V_{Q}^{-1},
\end{gathered}
$$


with common eigenvalue matrix

$$
D=\operatorname{diag}\left(e^{-i \pi \alpha}, e^{i \pi \alpha}, e^{-i \pi \alpha}, e^{i \pi \alpha}\right),
$$

and where the eigenvector matrices $V_{P}$ and $V_{Q}$ can be chosen as

$$
V_{P}=I+\frac{1}{2 i \sin (\pi \alpha)}\left(E_{21}+e^{-i \pi \alpha} E_{23}+E_{43}\right), \quad V_{Q}=I+\frac{1}{2 i \sin (\pi \alpha)} E_{21} .
$$

On account of the spectral decompositions (5.73)-(5.74), the equation (5.72) can be rewritten as $D\left(V_{Q}^{-1} S_{1} V_{P}\right)=\left(V_{Q}^{-1} S_{1} V_{P}\right) D$, which allows the solution $S_{1}=V_{Q} V_{P}^{-1}$. So we can take

$$
S_{1}=V_{Q} V_{P}^{-1}=I-\frac{1}{2 i \sin (\pi \alpha)}\left(e^{-i \pi \alpha} E_{23}+E_{43}\right) .
$$

The constant matrices $S_{2}, S_{3}$ and $S_{4}$ are then obtained recursively from $S_{1}$ and (5.71), which now read

$$
\begin{aligned}
& S_{2}=I+\frac{1}{2 i \sin (\pi \alpha)}\left(E_{13}-e^{-i \alpha \pi} E_{23}-E_{43}\right), \\
& S_{3}=I-\frac{1}{2 i \sin (\pi \alpha)}\left(E_{13}+e^{i \pi \alpha} E_{23}+E_{43}\right), \\
& S_{4}=I-\frac{1}{2 i \sin (\pi \alpha)}\left(e^{i \pi \alpha} E_{23}+E_{43}\right) .
\end{aligned}
$$

With $S_{k}, k=1, \ldots, 4$, given in (5.76)-(5.79), we conclude that $P$ defined in (5.70) indeed satisfies the items (1)-(4) in the $\mathrm{RH}$ problem for $P(z)$ stated above.

The above description was for Case II. For Cases I and III similar constructions can be given. The key point is that in each case, the cyclic products of jump matrices in the left hand sides of (5.73) and (5.74), respectively, allow spectral decompositions with common eigenvalue matrix (5.75). We leave these constructions to interested readers.

Combining the results in this subsection and Section 5.6.2, we take $P=Q S$ as the parametrix in $B_{\delta}$ for Case II, where $S=I$ if $\alpha \geq 0$, and $S$ is given by (5.69), if $-1<\alpha<0$.

\subsection{Final transformation}

Using the local parametrices $P, P^{\text {Airy }}$ and the global parametrix $N_{\alpha}$, we define the final transformation as follows

$$
R(z)= \begin{cases}T(z)\left(P^{\text {Airy }}(z)\right)^{-1}, & \text { in the disks around }\left\{p, q,-r_{1},-r_{3}\right\} \backslash\{0\}, \\ T(z)(P(z))^{-1}, & \text { in the disk } B_{\delta} \text { around the origin, } \\ T(z)\left(N_{\alpha}(z)\right)^{-1}, & \text { elsewhere. }\end{cases}
$$

From our construction of the parametrices, it follows that $R$ satisfies the following RH problem: 


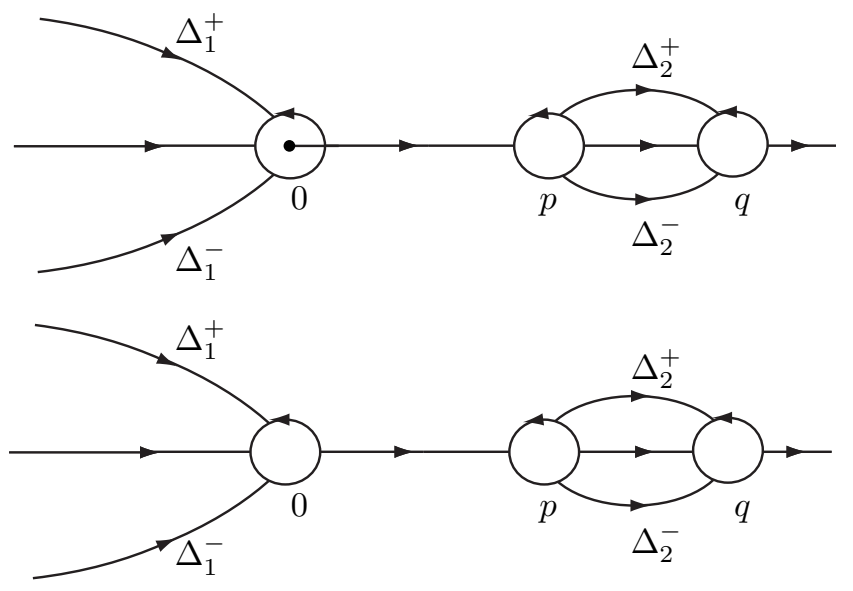

Figure 8: Jump contours $\Sigma_{R}$ for the RH problem for $R$ in Case I, for $\alpha \geq 0$ (top) and for $-1<\alpha<0$ (bottom).

(1) $R$ is analytic in $\mathbb{C} \backslash \Sigma_{R}$, where $\Sigma_{R}$ depends on $\alpha$ and is different for all three cases. For Cases I and III, an illustration is shown in Figures 8 and 9, respectively.

(2) $R$ has jumps $R_{+}=R_{-} J_{R}$ on $\Sigma_{R}$ that satisfy

$$
J_{R}(z)=I+O(1 / n),
$$

uniformly for $z$ on the boundaries of the disks;

$$
J_{R}(x)=I+O\left(x^{\alpha} e^{-c n}\right),
$$

on $(0, \delta)$ (Case I), or on $(-\delta, 0) \cup(0, \delta)$ (Case II), or on $(-\delta, 0)$ (Case III), for some constant $c>0$, if $\alpha \geq 0$

$$
J_{R}(z)=I+O\left(e^{-c n|z|}\right),
$$

on the other parts of $\Sigma_{R}$, for some constant $c>0$.

(3) $R(z)=I+O(1 / z)$ as $z \rightarrow \infty$.

Then, as in $[16,17,38]$, we may conclude that

$$
R(z)=I+O\left(\frac{1}{n(|z|+1)}\right),
$$

as $n \rightarrow \infty$, uniformly for $z$ in the complex plane outside of $\Sigma_{R}$. The convergence holds in fact uniformly up to $\Sigma_{R}$, as long as we stay away from the points of self-intersection of $\Sigma_{R}$ (including the origin). 


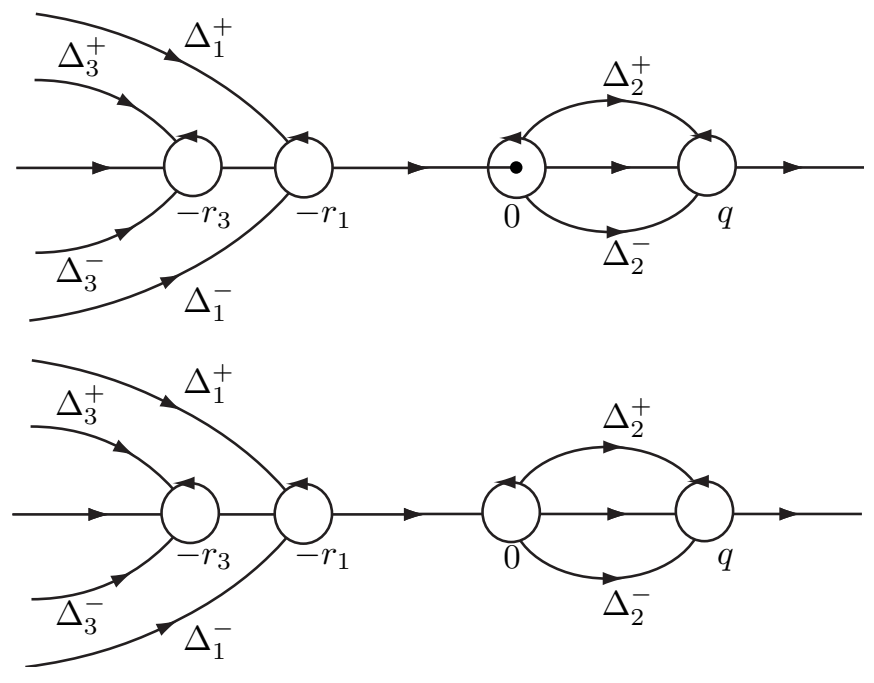

Figure 9: Jump contours $\Sigma_{R}$ for the RH problem for $R$ in Case III, for $\alpha \geq 0$ (top) and for $-1<\alpha<0$ (bottom).

\section{$6 \quad$ Proof of Theorem 2.3}

With the above preparations, we are ready to establish Theorem 2.3. For this purpose, we need to represent the correlation kernel $K_{n}(x, y)$ in terms of $T$ by following the sequence of transformations $Y \mapsto X \mapsto U \mapsto T$ in the RH analysis.

Let $x, y \in \Delta_{2}$. Recalling the representation of $K_{n}(x, y)$ in (2.34), we first find from (5.7) that

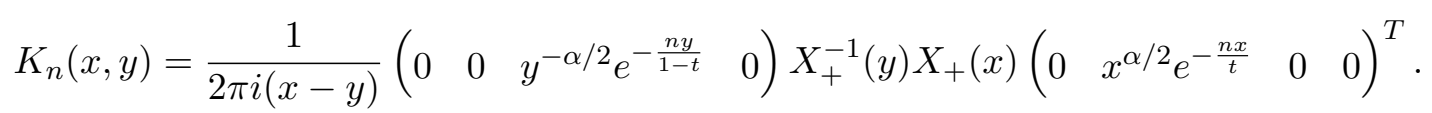

In view of (5.18), this becomes

$$
K_{n}(x, y)=\frac{x^{\alpha / 2} y^{-\alpha / 2}}{2 \pi i(x-y)}\left(\begin{array}{llll}
0 & 0 & e^{n \lambda_{3,-}(y)} & 0
\end{array}\right) U_{+}^{-1}(y) U_{+}(x)\left(\begin{array}{llll}
0 & e^{-n \lambda_{2,+}(x)} & 0 & 0
\end{array}\right)^{T} .
$$

By (5.38), it follows that

$$
\begin{aligned}
K_{n}(x, y)=\frac{x^{\alpha / 2} y^{-\alpha / 2}}{2 \pi i(x-y)}\left(\begin{array}{llll}
0 & -e^{n \lambda_{2,-}(y)} & e^{n \lambda_{3,-}(y)} & 0
\end{array}\right) T_{+}^{-1}(y) T_{+}(x) \\
\times\left(\begin{array}{llll}
0 & e^{-n \lambda_{2,+}(x)} & e^{-n \lambda_{3,+}(x)} & 0
\end{array}\right)^{T} .
\end{aligned}
$$

Now, we obtain from (5.84) and standard arguments (e.g. [7, Section 9]) that

$$
T_{+}^{-1}(y) T_{+}(x)=I+O(x-y), \quad \text { as } x \rightarrow y,
$$


uniformly in $n$. Also note that $\lambda_{3, \pm}=\lambda_{2, \mp}$. Thus, by taking $y \rightarrow x$, it follows from (6.2) and L'Hôpital's rule that

$$
K_{n}(x, x)=\frac{n}{2 \pi i}\left(\xi_{2,+}(x)-\xi_{2,-}(x)\right)+O(1)=n \frac{d \mu_{2}}{d x}(x)+O(1),
$$

where the last equality follows from (4.15), and so

$$
\lim _{n \rightarrow \infty} \frac{1}{n} K_{n}(x, x)=\frac{d \mu_{2}}{d x}(x), \quad x \in \Delta_{2} .
$$

If $x \in \mathbb{R}^{+} \backslash \Delta_{2}$, a similar argument as given above yields

$$
\lim _{n \rightarrow \infty} \frac{1}{n} K_{n}(x, x)=0 .
$$

This completes the proof of Theorem 2.3.

\section{Acknowledgement}

Steven Delvaux and Lun Zhang are Postdoctoral Fellows of the Fund for Scientific Research - Flanders (Belgium).

Arno Kuijlaars is supported by K.U. Leuven research grant OT/08/33, FWO-Flanders projects G.0427.09 and G.0641.11, by the Belgian Interuniversity Attraction Pole P06/02, and by grant MTM2008-06689-C02-01 of the Spanish Ministry of Science and Innovation.

Pablo Román is supported by K.U. Leuven research grant OT/08/33.

Lun Zhang is supported by the Belgian Interuniversity Attraction Pole P06/02.

\section{References}

[1] M. Abramowitz and I. A. Stegun, Handbook of Mathematical Functions with Formulas, Graphs, and Mathematical Tables, Dover Publications Inc., New York, 1992.

[2] M. Adler, P. L. Ferrari, and P. van Moerbeke, Non-intersecting random walks in the neighborhood of a symmetric tacnode. (arXiv:1007.1163).

[3] C. Álvarez-Fernández, U. Fidalgo Prieto, and M. Mañas, Multiple orthogonal polynomials of mixed type: Gauss-Borel factorization and the multi-component $2 D$ Toda hierarchy, Adv. Math. 227 (2011), 1451-1525.

[4] A. I. Aptekarev, P. M. Bleher, and A. B. J. Kuijlaars, Large $n$ limit of Gaussian random matrices with external source. II, Comm. Math. Phys. 259 (2005), 367-389.

[5] A.I. Aptekarev, V.A. Kalyagin, V. G. Lysov and D. N. Tulyakov, Equilibrium of vector potentials and uniformization of the algebraic curves of genus 0 , J. Comp. Appl. Math. 233 (2009), 602-616. 
[6] J. Baik, T. Kriecherbauer, K. T-R. McLaughlin, and P. D. Miller, Discrete orthogonal polynomials, vol. 164 of Annals of Mathematics Studies, Princeton University Press, Princeton, NJ, 2007.

[7] P. M. Bleher and A. B. J. Kuijlaars, Large $n$ limit of Gaussian random matrices with external source. I, Comm. Math. Phys. 252 (2004), 43-76.

[8] P. M. Bleher and A. B. J. Kuijlaars, Large $n$ limit of Gaussian random matrices with external source. III. Double scaling limit, Comm. Math. Phys. 270 (2007), 481-517.

[9] A. Borodin, Biorthogonal ensembles, Nuclear Phys. B 536 (1999), 704-732.

[10] A. Borodin and J. Kuan, Random surface growth with a wall and Plancherel measure for $O(\infty)$, Comm. Pure Appl. Math. 63 (2010), 831-894.

[11] A. N. Borodin and P. Salminen, Handbook of Brownian Motion-Facts and Formulae, Probability and its Applications, Birkhäuser Verlag, Basel, 1996.

[12] E. Brézin and S. Hikami, Universal singularity at the closure of a gap in a random matrix theory, Phys. Rev. E 57 (1998), 4140-4149.

[13] E. Daems and A. B. J. Kuijlaars, Multiple orthogonal polynomials of mixed type and non-intersecting Brownian motions, J. Approx. Theory 146 (2007), 91-114.

[14] E. Daems, A. B. J. Kuijlaars, and W. Veys, Asymptotics of non-intersecting Brownian motions and a $4 \times 4$ Riemann-Hilbert problem, J. Approx. Theory 153 (2008), $225-256$.

[15] P. Deift, Orthogonal polynomials and random matrices: a Riemann-Hilbert approach, vol. 3 of Courant Lecture Notes in Mathematics, New York University Courant Institute of Mathematical Sciences, New York, 1999.

[16] P. Deift, T. Kriecherbauer, K. T-R. McLaughlin, S. Venakides, and X. Zhou, Strong asymptotics of orthogonal polynomials with respect to exponential weights, Comm. Pure Appl. Math. 52 (1999), 1491-1552.

[17] P. Deift, T. Kriecherbauer, K. T-R. McLaughlin, S. Venakides, and X. Zhou, Uniform asymptotics for polynomials orthogonal with respect to varying exponential weights and applications to universality questions in random matrix theory, Comm. Pure Appl. Math. 52 (1999), 1335-1425.

[18] S. Delvaux and A. B. J. Kuijlaars, A phase transition for non-intersecting Brownian motions, and the Painlevé II equation, Int. Math. Res. Not. (2009), 3639-3725.

[19] S. Delvaux, A. B. J. Kuijlaars, and L. Zhang, Critical behavior of non-intersecting Brownian motions at a tacnode, Comm. Pure Appl. Math. 64 (2011), 1305-1383.

[20] P. Desrosiers and P. J. Forrester, A note on biorthogonal ensembles, J. Approx. Theory 152 (2008), 167-187. 
[21] P. D. Dragnev and E. B. Saff, Constrained energy problems with applications to orthogonal polynomials of a discrete variable, J. Anal. Math. 72 (1997), 223-259.

[22] M. Duits and D. Geudens, Double scaling limit for the two-matrix model in the quartic-quadratic case, in preparation.

[23] M. Duits and A. B. J. Kuijlaars, Universality in the two-matrix model: a RiemannHilbert steepest-descent analysis, Comm. Pure Appl. Math. 62 (2009), 1076-1153.

[24] M. Duits, A. B. J. Kuijlaars, and M. Y. Mo, The Hermitian two-matrix model with an even quartic potential, to appear in Memoirs Amer. Math. Soc. (arXiv:1010.4282)

[25] H. M. Farkas and I. Kra, Riemann surfaces, Graduate Texts in Mathematics 71, Springer-Verlag, New York-Berlin, 1980.

[26] P. J. Forrester, Log-Gases and Random Matrices, Princeton University Press, Princeton N.J., 2010.

[27] A. A. Gonchar and E. A. Rakhmanov, On the convergence of simultaneous Padé approximants for systems of Markov type functions, Proc. Stekl. Math. Inst. 3 (1983), $31-50$.

[28] A. A. Gonchar and E. A. Rakhmanov, The equilibrium problem for vector potentials, Uspekhi Mat. Nauk 40 (1985), 155-156 (in Russian).

[29] A. Hardy and A. B. J. Kuijlaars, Weakly admissible vector equilibrium problems, arXiv: 1110.6800 .

[30] I. Karatzas and S. Shreve, Brownian motion and stochastic calculus, Graduate Texts in Mathematics 113, Springer-Verlag, New York-Berlin, 1988.

[31] S. Karlin and J. McGregor, Coincidence properties of birth and death processes, Pacific J. Math. 9 (1959), 1109-1140.

[32] M. Katori, Determinantal processes starting from GOE-type eigenvalue distributions are Pfaffian processes. (arXiv 1104.1864).

[33] M. Katori, M. Izumi, and N. Kobayashi, Two Bessel bridges conditioned never to collide, double Dirichlet series, and Jacobi theta function, J. Stat. Phys. 131 (2008), 1067-1083.

[34] M. Katori and H. Tanemura, Symmetry of matrix-valued stochastic processes and noncolliding diffusion particle systems, J. Math. Phys. 45 (2004), 3058-3085.

[35] M. Katori and H. Tanemura, Noncolliding squared Bessel processes, J. Stat. Phys. 142 (2011), 592-615.

[36] W. König and N. O'Connell, Eigenvalues of the Laguerre process as non-colliding squared Bessel processes, Electron. Comm. Probab. 6 (2001), 107-114. 
[37] A. B. J. Kuijlaars and P. D. Dragnev, Equilibrium problems associated with fast decreasing polynomials, Proc. Amer. Math. Soc. 127 (1999), 1065-1074.

[38] A. B. J. Kuijlaars, A. Martínez-Finkelshtein, and F. Wielonsky, Non-intersecting squared Bessel paths and multiple orthogonal polynomials for modified Bessel weights, Comm. Math. Phys. 286 (2009), 217-275.

[39] A. B. J. Kuijlaars, A. Martínez-Finkelshtein, and F. Wielonsky, Non-intersecting squared Bessel paths: critical time and double scaling limit, to appear in Comm. Math. Phys. (arXiv:1011.1278)

[40] A. B. J. Kuijlaars, K. T-R. McLaughlin, W. Van Assche, and M. Vanlessen, The Riemann-Hilbert approach to strong asymptotics for orthogonal polynomials on $[-1,1]$, Adv. Math. 188 (2004), 337-398.

[41] A. B. J. Kuijlaars and M. Y. Mo, The global parametrix in the Riemann-Hilbert steepest descent analysis for orthogonal polynomials, Comput. Meth. Funct. Theory 11 (2011), 161-178.

[42] A. B. J. Kuijlaars and P. Román, Recurrence relations and vector equilibrium problems arising from a model of non-intersecting squared Bessel paths, J. Approx. Theory 162 (2010), 2048-2077.

[43] V. Lysov and F. Wielonsky, Strong asymptotics for multiple Laguerre polynomials, Constr. Approx. 28 (2008), 61-111.

[44] M. L. Mehta, Random Matrices, 3rd edition, Elsevier/Academic Press, Amsterdam, 2004.

[45] R. Miranda, Algebraic Curves and Riemann Surfaces, vol. 5 of Graduate Studies in Mathematics, American Mathematical Society, Providence, RI, 1995.

[46] E. M. Nikishin and V. N. Sorokin, Rational Approximations and Orthogonality, vol. 92 of Translations of Mathematical Monographs, American Mathematical Society, Providence, RI, 1991.

[47] E.A. Rakhmanov, Equilibrium measure and the distribution of zeros of the extremal polynomials of a discrete variable, Sb. Math. 187 (1996), 1213-1228.

[48] E. B. Saff and V. Totik, Logarithmic Potentials with External Fields, SpringerVerlag, Berlin, 1997.

[49] C. A. Tracy and H. Widom, Nonintersecting Brownian excursions, Ann. Appl. Probab. 17 (2007), 953-979.

[50] W. Van Assche, J. S. Geronimo, and A. B. J. Kuijlaars, Riemann-Hilbert problems for multiple orthogonal polynomials, in Special functions 2000: current perspective and future directions (J. Bustoz et al., eds.), Kluwer Acad. Publ., Dordrecht, 2001, pp. $23-59$. 